\title{
Comparison and rigidity theorems in semi-Riemannian geometry
}

\author{
LARS ANDERSSON ${ }^{1}$ AND RALPH HOWARD ${ }^{2}$
}

\begin{abstract}
The comparison theory for the Riccati equation satisfied by the shape operator of parallel hypersurfaces is generalized to semiRiemannian manifolds of arbitrary index, using one-sided bounds on the Riemann tensor which in the Riemannian case correspond to one-sided bounds on the sectional curvatures. Starting from 2-dimensional rigidity results and using an inductive technique, a new class of gap-type rigidity theorems is proved for semiRiemannian manifolds of arbitrary index, generalizing those first given by Gromov and Greene-Wu. As applications we prove rigidity results for semi-Riemannian manifolds with simply connected ends of constant curvature.
\end{abstract}

\section{Contents.}

1. Introduction

1.1. Background

1.2. Overview of this paper

\section{Preliminaries}

2.1. Basic Formulas and Results

2.2. Warped Products and Model Spaces

2.3. Two Dimensional Rigidity Results

\footnotetext{
${ }^{1}$ Supported in part by NFR, contract no. F-FU 4873-307.

${ }^{2}$ Supported in part by NFR, contract no. R-RA 4873-306 and the Swedish Academy of Sciences.
} 
3. Basic Comparison Results

3.1. Comparisons for Parallel Hypersurfaces

3.2. Comparisons for Tubes

4. Rigidity in Flat Spaces

5. Rigidity in Simply Connected Space Forms

5.1. Rigidity Lemmas in Warped Products

5.2. The Rigidity Results

5.3. Examples

6. Applications

6.1. Ends of Constant Curvature and Rigidity

6.2. Rigidity in Quotients

References

\section{Introduction.}

In Riemannian geometry, the comparison results in terms of sectional curvature of Rauch, Toponogov, Morse-Schoenberg and others (cf. [7]) are basic tools used to prove results such as the sphere theorem, the BonnetMyers theorem, and the maximal diameter theorem of Toponogov. More recently, comparison theorems in terms of the Ricci curvature such as the Bishop-Gromov volume comparison theorem have played an important role leading to such results as the Chen maximal diameter theorem, for example see the wonderful survey article by Karcher [23].

In Lorentzian geometry and semi-Riemannian geometry, on the other hand, it is well known (cf. [17] and Section 5.3 for a discussion) that the assumption of even a one-sided bound on sectional curvature defined as

$$
\frac{\langle R(X, Y) Y, X\rangle}{\langle X, X\rangle\langle Y, Y\rangle-\langle X, Y\rangle^{2}},
$$

implies that the space has constant sectional curvature. Therefore such bounds are not interesting.

In the general semi-Riemannian setting the natural replacement of a one side bound on the sectional curvature is 
Definition 1.1. We will say that $R \geq K_{0}$ or $R \leq K_{0}$ if and only if for all $X, Y$, the inequalities

$$
\langle R(X, Y) Y, X\rangle \geq K_{0}\left(\langle X, X\rangle\langle Y, Y\rangle-\langle X, Y\rangle^{2}\right),
$$

or

$$
\langle R(X, Y) Y, X\rangle \leq K_{0}\left(\langle X, X\rangle\langle Y, Y\rangle-\langle X, Y\rangle^{2}\right)
$$

hold.

In case $g$ is positive definite these conditions are equivalent to $g$ having sectional curvature bounded from below or above by $K_{0}$. Assuming bounds of the form given in this definition we are able to prove "gap type" rigidity results of the type first proved by Greene and Wu [16] and Gromov [1]. Our main example of such a result is

Theorem 1.2. Let $(M, g)$ be a geodesically complete semi-Riemannian manifold of dimension $n \geq 3$ and index $k$ with curvature satisfying one of the two inequalities $R \geq 0$ or $R \leq 0$ and assume $(M, g)$ has an end $E$ with $R \equiv 0$ on $E$ and $\pi_{1}(E)$ finite. Then $(M, g)$ is isometric to the flat model space $\left(\mathbb{R}_{k}^{n}, g_{0}\right)$.

In the Lorentzian and Riemannian case we also have rigidity results for comparison with constant, but non-zero, curvature models, cf. Section 5.2. Even in the Riemannian case this leads to a new rigidity result.

Theorem 1.3. Let $(M, g)$ be a complete Riemannian manifold of dimension $n \geq 3$ with sectional curvatures $\leq 1$. Let $B \subset S^{n} \backslash S^{n-1}$ be a closed set with $S^{n} \backslash B$ connected and let $\varphi: S^{n} \backslash B \rightarrow M$ be a local isometry. Then $\varphi$ extends to a surjective local isometry $\widehat{\varphi}: S^{n} \rightarrow M$. Therefore $(M, g)$ is a quotient of $S^{n}$ by a finite fixed point free group of isometries.

This result differs from most rigidity theorems for the sphere in that an upper bound, rather than a lower bound, is given on the curvature.

The proofs of the gap theorems rest on two main ideas. The first is an extension of the comparison theory for matrix Riccati equations to the semidefinite setting, cf. Section 3. The second is an inductive argument involving the use of foliations by parallel hypersurfaces, with base case given by some 2-dimensional rigidity results, see Section 2.3. The two are related by the fact that the second fundamental form of a parallel foliation satisfies the Riccati equation. 


\subsection{Background.}

One important class of results in Riemannian geometry are the "gap type" rigidity theorems. Let us mention a few examples of model gap results. We do not try to state these results under the weakest hypotheses. Let $(M, g)$ be a complete connected Riemannian manifold, isometric to Euclidean $\mathbb{R}^{n}$ in the complement of a compact set with $n \geq 3$.

- Scalar curvature: Assume that $(M, g)$ is spin and has non-negative scalar curvature. Then $(M, g)$ is isometric to Euclidean $\mathbb{R}^{n}$. This follows from the Witten argument for the positive mass theorem.

- Ricci curvature: Assume that $(M, g)$ has non-negative Ricci curvature. Then the conclusion follows from the Bishop-Gromov volume comparison theorem.

- Sectional curvature: If $(M, g)$ has either non-negative or nonpositive sectional curvature, then theorems of Greene and Wu [16] (for non-negative sectional curvature) and Kasue and Sugahara [24] (for non-positive sectional curvature) imply $(M, g)$ is isometric to $\mathbb{R}^{n}$.

In each of the above cases there are versions where the assumption that $(M, g)$ is isometric to the standard space in the complement of a compact set is replaced by asymptotic assumptions. There are also versions where the Euclidean space is replaced by constant curvature or homogeneous spaces. Cf. Sections 4, 5.2, 6.1, and 6.2 for further discussion.

In the Lorentzian case, the most important rigidity type result is of course the positive mass theorem of Schoen-Yau and Witten (cf. [33, 39, 30]). Witten's proof has been generalized to the case of the Bondi mass by various authors [21], [31], [28].

In the Riemannian case, the dependence of the scalar curvature on $g$ can be viewed as a scalar elliptic operator acting on the conformal factor, and the Ricci tensor can be viewed as a quasilinear elliptic system in terms of the metric tensor. This makes the form of the gap type rigidity theorems natural, in view of the maximum principle and unique continuation results which hold for elliptic equations.

In the Lorentzian case, the Ricci and Einstein tensors form hyperbolic systems in terms of the metric tensor and the positive mass theorems is closely related to the elliptic constraints induced via the Gauss and GaussCodazzi equations on the extrinsic geometry of spacelike hypersurfaces. Further, in this case, causality is a powerful organizing principle in the geometry 
which shows up for example in the conservation theorem $[18, \S 4.3]$ and Theorem 5.13 below.

From the PDE point of view, putting conditions on the whole Riemann tensor constitutes an over determined system, regardless of the signature. More precisely in normal coordinates centered at a point of a semiRiemannian manifold it is possible to express all the derivatives of order at least two of the metric in terms of the curvature tensor and its derivatives. Thus the operator that takes a metric to its curvature tensor behaves very much like an elliptic operator in that it is possible to bound the second and higher derivatives of the unknown metric in terms of the curvature tensor. Therefore, one expects bounds on the Riemann tensor to give gap type rigidity theorems regardless of the signature, as is shown to be the case in the present paper.

In the Lorentzian case, the one-sided curvature bounds used in this paper implies conditions on the timelike sectional curvatures in the sense of [3]. For example, $R \geq 0$, implies nonpositive timelike sectional curvatures in the sense of [3]. The condition $R \geq 0$ also implies the strong energy condition, i.e. that $\operatorname{Ric}(X, X) \geq 0$ for timelike vectors $X$. In this situation, the Lorentzian splitting theorems of Galloway, Eschenburg, Newman and others [13] would apply if we add the condition of existence of a timelike ray in the future of a maximal hypersurface, cf. [12, Theorem C]. This would then easily imply the Lorentzian case of Theorem 4.2. Therefore it is important that our assumptions do not include the existence of a timelike ray.

Further, in view of the fact that $R \geq 0$ implies the strong energy condition, under the additional assumption of global hyperbolicity, Theorem 4.2 would in the Lorentzian case follow easily from the singularity theorems in Lorentzian geometry, cf. [18]. Therefore we also stress that our conditions do not include global hyperbolicity.

Let us emphasize that the conditions of timelike geodesic completeness and global hyperbolicity are not at all closely related. As an illustration of this fact, let us mention that anti-de Sitter space is timelike geodesically complete but not globally hyperbolic, while the "strip" $\mathbf{S}_{1}^{n}(-1)$, cf. Definition 2.6 , which is a subset of anti-de Sitter space, is globally hyperbolic, but not timelike geodesically complete. The assumption of timelike geodesic completeness is thus natural in the context of anti-de Sitter space.

\subsection{Overview of this paper.}

Section 2 contains the basic results and formulas we will be using. Almost all of this is well known, but is included to fix notation and as the details of 
writing the Riemannian and Lorentzian space forms as warped products is quite important to many of our rigidity results.

Section 3 contains the generalization of the basic comparison theory to the general semi-Riemannian setting. Most of the results are stated as results about systems of ordinary differential equations.

Section 4 gives the rigidity results for flat semi-Riemannian manifolds in terms of one sided bounds on the curvature tensor.

Section 5 has rigidity results for the one sided bounds on the sectional curvature when the model space has non-zero constant sectional curvature. The results in this section only apply to Riemannian and Lorentzian manifolds.

Section 6 contains various applications of the rigidity results to manifolds that have an end of constant curvature. This involves a classification of the ends of constant curvature and finite fundamental groups and which is new in the case of indefinite metrics. There are also rigidity results for semiRiemannian metrics on quotients of space forms.

Acknowledgements. E. Calabi [6] supplied us with Theorem 2.12 which replaces a weaker ad hoc version. Much of the work on this paper was done while the second author $(\mathrm{RH})$ was on sabbatical leave from the University of South Carolina at the Royal Institute of Technology in Stockholm. He would like to express his thanks to both schools.

\section{Preliminaries.}

\subsection{Basic Formulas and Results.}

The index of a symmetric bilinear form $g($,$) is the maximal dimension$ of a subspace on which it is negative definite, or equivalently, the number of negative eigenvalues. If $\bar{M}$ is a smooth manifold then a semi-Riemannian metric $\bar{g}$ on $\bar{M}$ is a smooth $(0,2)$ tensor which is everywhere non-degenerate. The index of $\bar{g}$ is locally constant so if $\bar{M}$ is connected then the index $k$ of $\bar{g}$ is well defined. Thus the semi-Riemannian manifolds of index 0 are the Riemannian manifolds and the ones of index 1 are the Lorentzian manifolds. Unlike the Riemannian case not every manifold need have a semiRiemannian metric of index $k$. A necessary and sufficient condition for a smooth manifold $\bar{M}$ to have a semi-Riemannian metric of index $k$ is that the 
tangent bundle $T(\bar{M})$ have a rank $k$ subbundle $E$. For $\bar{M}$ compact this is proven in [36, Theorem 40.11], but the extension to noncompact manifolds is easy. In particular, every noncompact manifold has a Lorentzian metric.

Let $\bar{M}$ be an $n$ dimensional semi-Riemannian manifold with semiRiemannian metric $\bar{g}()=,\langle$,$\rangle of index k$. An immersed submanifold $M$ of $\bar{M}$ of dimension $m$ is called non-degenerate if the restriction of the semiRiemannian metric $\langle$,$\rangle to T(M)$ is non-degenerate at each point of $M$. The normal bundle to $M$ in $\bar{M}$ will be denoted by $T^{\perp}(M)$. This is the bundle over $M$ so that at each $x \in M$ the fiber $\left\{Y \in T(\bar{M})_{x}:\langle Y, X\rangle=0\right.$ for all $X \in$ $\left.T(M)_{x}\right\}$. There is a direct sum decomposition

$$
T(\bar{M})_{x}=T(M)_{x} \oplus T^{\perp}(M)_{x}
$$

at a point $x \in M$ if and only if the restriction of $\langle$,$\rangle to T(M)_{x}$ is nondegenerate. This also equivalent to the restriction of $\langle$,$\rangle to the normal$ bundle $T^{\perp}(M)$ being non-degenerate. For the rest of this section we will assume that the induced metric on $M$ is non-degenerate.

Let $\bar{\nabla}$ be the metric connection on $\bar{M}^{n}$ and let $\nabla$ be the metric connection on $M$. These are related by

$$
\bar{\nabla}_{X} Y=\nabla_{X} Y+h(X, Y)
$$

where $X, Y$ are smooth vector fields tangent to $M$, and $h($,$) is the vector$ valued second fundamental form of $M$ in $\bar{M}$. Thus $h($,$) is a symmetric$ bilinear map from $T(M) \times T(M) \rightarrow T^{\perp}(M)$. If $\xi$ is a smooth section of $T^{\perp}(M)$ over $M$ and $X$ is a smooth vector field tangent to $M$ then

$$
\bar{\nabla}_{X} \xi=\nabla_{X}^{\frac{1}{X}} \xi-A_{\xi} X
$$

where $\nabla_{X}^{\perp} \xi \in T^{\perp}(M)$ and $A_{\xi} X \in T(M)$. Then $\nabla^{\perp}$ is the connection in the normal bundle, and $A_{\xi}$ is the Weingarten map or shape operator in the normal direction $\xi$. This is related to the second fundamental form by

$$
\left\langle A_{\xi} X, Y\right\rangle=\langle h(X, Y), \xi\rangle .
$$

Thus the symmetry of $h($,$) implies that for any \xi \in T^{\perp}(M)$ the linear map $A_{\xi}: T(M) \rightarrow T(M)$ is self-adjoint with respect to the inner product $\langle$,$\rangle .$

Let $\bar{R}$ be the curvature tensor of $\bar{\nabla}$ where our choice of sign on the curvature tensor is

$$
\bar{R}(X, Y) Z=\bar{\nabla}_{X} \bar{\nabla}_{Y} Z-\bar{\nabla}_{Y} \bar{\nabla}_{X} Z-\bar{\nabla}_{[X, Y]} Z .
$$


This is related to the curvature tensor of $\nabla$ by the Gauss curvature equation

$$
\begin{aligned}
\langle R(X, Y) Z, W\rangle=\langle\bar{R}(X, Y) Z, W\rangle & \\
& +\langle h(X, W), h(Y, Z)\rangle-\langle h(X, Z), h(Y, W)\rangle .
\end{aligned}
$$

When $M$ is a hypersurface with unit normal field $\xi$ this can be rewritten in terms of the Weingarten map as

$$
\begin{aligned}
\langle R(X, Y) Z, W\rangle & =\langle\bar{R}(X, Y) Z, W\rangle \\
& +\langle\xi, \xi\rangle\left(\left\langle A_{\xi} X, W\right\rangle\left\langle A_{\xi} Y, Z\right\rangle-\left\langle A_{\xi} X, Z\right\rangle\left\langle A_{\xi} Y, W\right\rangle\right) .
\end{aligned}
$$

The normal bundle has two unit sphere bundles $S_{+1}^{\perp}(M)$ and $S_{-1}^{\perp}(M)$. Letting $\varepsilon$ be either $\varepsilon=+1$ or $\varepsilon=-1$ they are defined by

$$
S_{\varepsilon}^{\perp}(M)=\left\{u \in T^{\perp}(M):\langle u, u\rangle=\varepsilon\right\} .
$$

Each of these is a bundle $\pi: S_{\varepsilon}^{\perp}(M) \rightarrow M$ with fiber diffeomorphic to the "sphere" $\left\{u \in T^{\perp}(M)_{x}:\langle u, u\rangle=\varepsilon\right\}$.

Let $\pi: T^{\perp}(M) \rightarrow M$ be the projection and for $v \in T^{\perp}(M)$ let $\mathcal{V}_{v}:=$ $\operatorname{ker}\left(\pi_{* v}\right)$ be the vertical vectors at $v$. There is a natural identification of $\mathcal{V}_{v}$ with the subspace $T^{\perp}(M)_{\pi x}$ of $T(\bar{M})_{\pi v}$. In a vector space the tangent spaces $T(V)_{x}$ are naturally identified with $V$. The fibers $\pi^{-1}[x]=T^{\perp}(M)_{x}$ of $\pi$ are vector spaces and $\mathcal{V}_{v}=T^{\perp}(M)_{\pi x} \subset T(\bar{M})_{\pi v}$. Let $\mathcal{H}_{v}$ be the space of horizontal vectors at $v$. A curve $\gamma:(a, b) \rightarrow T^{\perp}(M)$ is horizontal iff it it is parallel along its projection $c=\pi \circ \gamma$. That is it satisfies $\nabla_{c^{\prime}(t)}^{\perp} \gamma=0$. A vector $X$ tangent to $T^{\perp}(M)$ is horizontal iff it is tangent to a horizontal curve. The space $\mathcal{H}_{v}$ can be identified with $T(M)_{\pi V}$ by the map $\pi_{*} \mid \mathcal{H}_{v}$. These identifications combine to give $T\left(T^{\perp}(M)\right)_{v}=\mathcal{H}_{v} \oplus \mathcal{V}_{v}=T(M)_{\pi v} \oplus$ $T^{\perp}(M)_{v}=T(\bar{M})_{\pi v}$. Under this identification for $u \in S_{\varepsilon}^{\perp}(M)$ the tangent space $T\left(S_{\varepsilon}^{\perp}\right)_{u}$ is identified with $u^{\perp} \subset T(M)_{\pi u}$.

Let exp be the exponential map defined w.r.t. $\bar{\nabla}$ and define a function $f_{r}: S_{\varepsilon}^{\perp} \rightarrow \bar{M}$ by $M(r):=f_{r}\left[S_{\varepsilon}^{\perp}\right]$. Then $M(r)$ is the tube of radius $r$ (and sign $\varepsilon$ ) about $M$. Let $u \in T(\bar{M})_{x}$ and let $\gamma(t)=\exp (t u)$ be the geodesic that fits $u$. Identify all the tangent spaces $T(\bar{M})_{\gamma(t)}$ along $\gamma$ with $T(\bar{M})_{x}$ by parallel translation along $\gamma$. For each $t$ let $\bar{R}_{u}(t): T(\bar{M})_{x} \rightarrow T(\bar{M})_{x}$ be the linear map

$$
\bar{R}_{u}(t) X=\bar{R}_{\gamma(t)}(X, u) u=\bar{R}_{\gamma(t)}\left(X, \gamma^{\prime}(t)\right) \gamma^{\prime}(t),
$$

where $\gamma^{\prime}(t)$ and $u$ are identified as $\gamma^{\prime}$ is parallel along $\gamma$. For each $t$ the linear map $\bar{R}_{u}(t)$ is self-adjoint (i.e. $\left.\left\langle\bar{R}_{u}(t) X, Y\right\rangle=\left\langle X, \bar{R}_{u}(t) Y\right\rangle\right)$. Also for each $t$, $\bar{R}_{u}(t) u=\bar{R}(u, u) u=0$. Thus from elementary linear algebra the subspace $u^{\perp}$ is invariant under $\bar{R}_{u}(t)$. 
Definition 2.1. For $u \in S_{\varepsilon}^{\perp}(M)$ let $P: u^{\perp} \rightarrow T(M)_{\pi u}$ and $P^{\perp}: u^{\perp} \rightarrow$ $T^{\perp}(M) \cap u^{\perp}$ be the orthogonal projections. Define a field of linear maps $F_{u}(t)$ along $\gamma(t)=\exp (t u)$ by the initial value problem:

$$
F_{u}^{\prime \prime}(t)+\bar{R}_{u}(t) F_{u}(t)=0, \quad F_{u}(0)=P, \quad F_{u}^{\prime}(0)=-A_{u} P+P^{\perp},
$$

where "prime" denotes the covariant derivative by $\gamma^{\prime}(t)\left(\right.$ i.e. $\left.{ }^{\prime}=\bar{\nabla}_{\gamma^{\prime}}\right)$ and $A_{u}$ is the Weingarten map of $M$ in the direction $u$.

Proposition 2.2. With the identification of $T\left(S_{\varepsilon}^{\perp}(M)\right)$ with $u^{\perp}$ given above, the derivative of $f_{r}$ at $u$ is

$$
f_{r * u}=F_{u}(r)
$$

Proof. Just as in the Riemannian case. Cf. [19].

If $F_{u}(r)$ is non-singular then the tube $M(r)$ is a smooth immersed hypersurface at $f_{r}(u)=\exp (r u)$. The outward normal at this point is

$$
\eta\left(f_{r}(u)\right)=\left.\frac{d}{d t} \exp (t u)\right|_{t=r} .
$$

At these smooth points of $M(r)$ the Weingarten map or of $M(r)$ with respect to this normal is the linear map $S_{u}(r): T(M(r))_{f_{r}(u)} \rightarrow T(M(r))_{f_{r}(u)}$ given by

$$
S_{u}(r) X=-\bar{\nabla}_{X} \eta
$$

Proposition 2.3. Identify $T(M(r))_{f_{r}(u)}$ with the subspace $u^{\perp}$ of $T(M)_{\pi u}$ by parallel translation along $t \mapsto \exp (t u)$. Then the Weingarten map is given by

$$
S_{u}(r)=-F_{u}^{\prime}(r) F_{u}(r)^{-1} .
$$

At points where $F_{u}(t)$ is non-singular this satisfies the Matrix Riccati equation

$$
S_{u}^{\prime}(t)=S_{u}(t)^{2}+\bar{R}_{u}(t)
$$

Proof. Follows from the last proposition by a calculation. Cf. [19]. 
The mean curvature of $M$ in the direction $\xi$ is

$$
H_{\xi}=\frac{1}{n-1} \operatorname{trace}\left(A_{\xi}\right) .
$$

and we denote by $H_{u}(r)$ the mean curvature of $M(r)$ at $f_{r}(u)$. If $A$ is an $(n-1) \times(n-1)$ symmetric matrix, then by the Cauchy-Schwartz inequality $(\operatorname{trace}(A))^{2} \leq(n-1) \operatorname{trace}\left(A^{2}\right)$ with equality if and only if $A=c I$ for some scalar $c$. This leads to:

Corollary 2.4. Assume that $\bar{M}$ is Riemannian or Lorentzian, and that $M$ is a space like hypersurface in $\bar{M}$ (so that the induced metric on $M(r)$ is Riemannian). Let $x \in M, \eta(x)$ the normal to $M$ at $x, S(t)$ the Weingarten map of $M(t)$ along $\gamma(t)=\exp (\eta(x))$ and $H(t)=\frac{1}{n-1} \operatorname{trace}(S(t))$ the mean curvature.

$$
H^{\prime}(t) \geq(n-1) H^{2}(t)+\operatorname{trace}\left(\bar{R}_{u}(t)\right)
$$

If equality holds at $t_{0}>0$ then $A_{\eta(x)}=S(0)=c I$ and $\bar{R}(t)=\bar{r}(t) I$ for $0 \leq t \leq t_{0}$.

A fact that will be used repeatedly in the sequel is that along $\gamma(t)=\exp (t u)$

$$
\overline{\operatorname{Ric}}\left(\gamma^{\prime}(t), \gamma^{\prime}(t)\right)=\operatorname{trace}\left(\bar{R}_{u}(t)\right) .
$$

We close this section with a result about when local isometries between semi-Riemannian manifolds are surjective.

Proposition 2.5. Let $M$ be a geodesically complete semi-Riemannian manifold and $f: M \rightarrow N$ a local isometry where $N$ is a connected semiRiemannian manifold of the same dimension as $M$. Then $f$ is surjective and $N$ is also geodesically complete.

Proof. As $f$ is a local diffeomorphism the image $f[M]$ is open. Thus it is enough to show that $f[M]$ is closed. Let $y$ be a point in the closure of $f[M]$. Then as the exponential map exp : $T N_{y} \rightarrow N$ maps a neighborhood of $0 \in T N_{y}$ onto a neighborhood of $y$ in $N$ there is a geodesic segment $c:[0, \delta] \rightarrow N$ so that $c(0)=y$ and $c(\delta) \in f[M]$. Using that $f$ is a local isometry we see there is a geodesic $\gamma: \mathbb{R} \rightarrow M$ so that $(f \circ \gamma)^{\prime}(0)=c^{\prime}(\delta)$. But then $f \circ \gamma: \mathbb{R} \rightarrow N$ is a geodesic and by the uniqueness of solutions to the geodesic equation we have $(f \circ \gamma)(-\delta)=c(0)=y$. Thus

$$
y=f(\gamma(-\delta)) \in f[M]
$$


Therefore $f[M]$ contains all its limit points and thus it is closed. This completes the proof that $f$ is surjective.

Let $c:(-\delta, \delta) \rightarrow N$ be a small geodesic segment. Then as $f$ is surjective and $M$ geodesically complete, there is a geodesic $\gamma: \mathbb{R} \rightarrow M$ so that $f \circ \gamma$ extends $c$ to all of $\mathbb{R}$. Thus $N$ is geodesically complete.

\subsection{Warped Products and Model Spaces.}

Let $K_{0} \in \mathbb{R}$ and let $k$ be an integer, $0 \leq k \leq n$. We will denote by $\mathbb{R}_{k}^{n}\left(K_{0}\right)$ the simply connected model space of sectional curvature $K_{0}$ and index $k$ and we will use $g_{\mathbb{R}_{k}^{n}\left(K_{0}\right)}$ to denote its metric. In the Riemannian case $(k=0)$ the model spaces are either Euclidean space, spheres or hyperbolic spaces. In the Lorentzian case, the non-flat model spaces are de Sitter $\left(K_{0}>0\right)$ and anti-de Sitter spaces $\left(K_{0}<0\right)$.

Let $n \geq 3$. Then $\mathbb{R}_{1}^{n}(1)$ is the hypersurface of $\mathbb{R}_{1}^{n+1}$ given by

$$
-x_{1}^{2}+\sum_{i=2}^{n+1} x_{i}^{2}=1,
$$

with its induced metric. This is known as de Sitter space and is diffeomorphic to $S^{n-1} \times \mathbb{R}$. Similarly, the hypersurface

$$
-x_{1}^{2}-x_{2}^{2}+\sum_{i=3}^{n+1} x_{i}^{2}=1
$$

of $\mathbb{R}_{2}^{n}$ with its induced metric has sectional curvature -1 and index 1 and is diffeomorphic to $S^{1} \times \mathbb{R}^{n-1}$. The universal cover of this space is known as anti-de Sitter space and will be denoted by $\mathbb{R}_{1}^{n}(-1)$.

Some of the model spaces we will consider are best represented as warped products of a hypersurface with the real line. For completeness we include the basic formulas in the geometry of warped products.

Let $M^{n-1}$ be a semi-Riemannian manifold with metric $g($,$) , and (a, b)$ be an open interval in $\mathbb{R}$, and $w$ a positive real valued function on $(a, b)$. Define a semi-Riemannian metric $\bar{g}_{w}($,$) on \bar{M}^{n}=M^{n-1} \times(a, b)$ by

$$
\bar{g}_{w}=w(t)^{2} g+\varepsilon(d t)^{2},
$$

where $\varepsilon=+1$ or $\varepsilon=-1$. A vector field $X$ on $M^{n-1}$ can also be viewed as a vector field on $\bar{M}$ in the natural way. Let $\nabla$ be the semi-Riemannian 
connection of $g$ and $\bar{\nabla}$ the semi-Riemannian connection of $\bar{g}_{w}$. If $X, Y$ are vector fields on $M$ then the connections $\nabla$ and $\bar{\nabla}$ are related by

$$
\bar{\nabla}_{X} Y=\nabla_{X} Y-\frac{\varepsilon w^{\prime} \bar{g}_{w}(X, Y)}{w} \partial_{t}, \quad \bar{\nabla}_{X} \partial_{t}=\bar{\nabla}_{\partial_{t}} X=\frac{w^{\prime}}{w} X, \quad \bar{\nabla}_{\partial_{t}} \partial_{t}=0 .
$$

The vector field $\eta:=\partial_{t}$ is a unit normal vector to the hypersurfaces $M^{n-1} \times$ $\{c\}$. From the above formulas the second fundamental form $h$ and the Weingarten map $S$ of these hypersurfaces are given by

$$
h(X, Y)=-\frac{\varepsilon w^{\prime}}{w} g_{w}(X, Y) \partial_{t}, \quad S X=-\frac{w^{\prime}}{w} X .
$$

The curvature $R$ of $M$ and the curvature $\bar{R}$ of $\bar{M}$ are related by

$$
\bar{R}\left(X, \partial_{t}\right) \partial_{t}=-\frac{w^{\prime \prime}}{w} X
$$

so that

$$
\bar{g}_{w}\left(\bar{R}\left(X, \partial_{t}\right) \partial_{t}, X\right)=-\frac{w^{\prime \prime}}{w} \bar{g}_{w}(X, X)=-\frac{\varepsilon w^{\prime \prime}}{w} \bar{g}_{w}(X, X) \bar{g}_{w}\left(\partial_{t}, \partial_{t}\right) .
$$

Therefore

$$
\operatorname{Ric}\left(\partial_{t}, \partial_{t}\right)=-(n-1) \frac{w^{\prime \prime}(t)}{w(t)} .
$$

Using the Gauss curvature equation (2.2) there is also the relation

$$
\begin{aligned}
\bar{g}_{w}(R(X, Y) Y, X) & =\bar{g}_{w}(\bar{R}(X, Y) Y, X) \\
& +\varepsilon\left(\frac{w^{\prime}}{w}\right)^{2}\left(\bar{g}_{w}(X, X) \bar{g}_{w}(Y, Y)-\bar{g}_{w}(X, Y)^{2}\right)
\end{aligned}
$$

If $(M, g)$ has constant sectional curvature $K_{0}$ then

$$
g(R(X, Y) Y, X)=K_{0}\left(g(X, X) g(Y, Y)-g(X, Y)^{2}\right),
$$

so that

$$
\bar{g}_{w}(R(X, Y) Y, X)=\left(\frac{K_{0}}{w^{2}}\right)\left(\bar{g}_{w}(X, X) \bar{g}_{w}(Y, Y)-\bar{g}_{w}(X, Y)^{2}\right) .
$$

Using this in equation (2.13) leads to

$$
\bar{g}_{w}(\bar{R}(X, Y) Y, X)=\frac{K_{0}-\varepsilon\left(w^{\prime}\right)^{2}}{w^{2}}\left(\bar{g}_{w}(X, X) \bar{g}_{w}(Y, Y)-\bar{g}_{w}(X, Y)^{2}\right)
$$


for vector fields $X, Y$ tangent to $M$.

The special cases we need are summarized in the following table. The first column gives the isometry class of the factor $M$, the next two columns give the warping function $w$ where the parameter $\alpha$ is defined by the equation in the second column. The metric $\bar{g}_{w}$ is then given by equation (2.11) with $\varepsilon$ as in the fourth column. The isometry class of the warped product $\bar{M}$ is then given in the fifth column. The last two columns give the isometry class of the hypersurface $M_{t}:=M \times\{t\}$ and the Weingarten map $S_{t}$ of $M_{t}$ with respect to the normal $\eta:=\partial_{t}$.

Table 1.

\begin{tabular}{|c|c|c|c|c|c|c|}
\hline$M$ & $\alpha$ & $w$ & $\varepsilon$ & $\bar{M}$ & $M_{t}$ & $S_{t}$ \\
\hline \hline $\mathbb{R}_{0}^{n-1}(0)$ & - & $e^{-t}$ & +1 & $\mathbb{R}_{0}^{n}(-1)$ & $\mathbb{R}_{0}^{n}(0)$ & $I$ \\
\hline $\mathbb{R}_{0}^{n-1}(0)$ & - & $e^{t}$ & -1 & $\begin{array}{c}\text { Part of } \\
\mathbb{R}_{1}^{n}(+1)\end{array}$ & $\mathbb{R}_{0}^{n-1}(0)$ & $-I$ \\
\hline $\begin{array}{c}\mathbb{R}_{0}^{n-1}\left(K_{0}\right), \\
K_{0} \geq 1\end{array}$ & $\begin{array}{c}\cos (\alpha) \\
=\frac{1}{\sqrt{K_{0}}}\end{array}$ & $\frac{\cos (t+\alpha)}{\cos (\alpha)}$ & +1 & $\mathbb{R}_{0}^{n}(+1)$ & $\mathbb{R}_{0}^{n-1}\left(\frac{+1}{\cos ^{2}(t+\alpha)}\right)$ & $\tan (t+\alpha) I$ \\
\hline $\begin{array}{c}\mathbb{R}_{0}^{n-1}\left(K_{0}\right), \\
-1 \leq K_{0}<0\end{array}$ & $\begin{array}{c}\cosh (\alpha) \\
=\frac{1}{\sqrt{\left|K_{0}\right|}}\end{array}$ & $\frac{\cosh (t+\alpha)}{\cosh (\alpha)}$ & +1 & $\mathbb{R}_{0}^{n}(-1)$ & $\mathbb{R}_{0}^{n-1}\left(\frac{-1}{\cosh ^{2}(t+\alpha)}\right)$ & $-\tanh (t+\alpha) I$ \\
\hline $\begin{array}{c}\mathbb{R}_{0}^{n-1}\left(K_{0}\right), \\
0<K_{0} \leq 1\end{array}$ & $\begin{array}{c}\cosh (\alpha) \\
=\frac{1}{\sqrt{K_{0}}}\end{array}$ & $\frac{\cosh (t+\alpha)}{\cosh (\alpha)}$ & -1 & $\mathbb{R}_{1}^{n}(+1)$ & $\mathbb{R}_{0}^{n-1}\left(\frac{1}{\cosh ^{2}(t+\alpha)}\right)$ & $-\tanh (t+\alpha) I$ \\
\hline $\begin{array}{c}\mathbb{R}_{0}^{n-1}\left(K_{0}\right) \\
K_{0} \leq-1\end{array}$ & $\begin{array}{c}\cos (\alpha) \\
=\frac{1}{\sqrt{\left|K_{0}\right|}}\end{array}$ & $\frac{\cos (t+\alpha)}{\cos (\alpha)}$ & -1 & $\begin{array}{l}P \operatorname{Part} \text { of } \\
\mathbb{R}_{1}^{n}(-1)\end{array}$ & $\mathbb{R}_{0}^{n-1}\left(\frac{-1}{\cos ^{2}(t+\alpha)}\right)$ & $\tan (t+\alpha) I$ \\
\hline
\end{tabular}

Two entries in this table require some explanation. The first is the second row which is the warped product of the Euclidean space $\mathbb{R}^{n-1}$ with its standard metric $g=|d x|^{2}$ and $w(t)=e^{-t}$ so that $\bar{g}_{w}=e^{-2 t}|d x|^{2}-d t^{2}$. If we do the change of variable $y=e^{-t}$, then the metric becomes

$$
\bar{g}_{w}=\frac{|d x|^{2}-d y^{2}}{y^{2}}
$$

defined on the upper half space

$$
\left\{(x, y) \in \mathbb{R}^{n-1} \times \mathbb{R}: y>0\right\}
$$

(where $|d x|^{2}:=d x_{1}^{2}+\cdots+d x_{n-1}^{2}$ is the standard metric on $\mathbb{R}^{n-1}$ ). This is diffeomorphic to $\mathbb{R}^{n}$ and thus can not be all of the de Sitter space $\mathbb{R}_{1}^{n}(+1)$ as de Sitter space is diffeomorphic to $S^{n-1} \times \mathbb{R}$. Therefore the metric $\bar{g}_{w}$ is not geodesically complete. (When $n=2$ this can be seen more concretely by noting that $c(t)=(\sinh (t), \cosh (t))$ is a geodesic in the upper half plane 
with the metric $\bar{g}_{w}$, but that the length of $c$ is $\int_{-\infty}^{\infty} \frac{d t}{\cosh (t)}=\pi<\infty$.) We also note that if $a \in \mathbb{R}^{n-1}$ and $c>0$ then the metric $\bar{g}_{w}$ is invariant under the transformations $(x, y) \mapsto(x+a, y)$ and $(x, y) \mapsto(c x, c y)$ and thus the upper half plane has a transitive group of isometries. Thus unlike the Riemannian case a semi-Riemannian homogeneous space need not be geodesically complete. It is convenient to give this half space part of the de Sitter space a name.

Definition 2.6. Denote by $\left(\mathbf{H}_{1}^{n}(+1), g_{\mathbf{H}}\right)$ the space $\mathbf{H}_{1}^{n}(+1)=\mathbb{R}^{n-1} \times \mathbb{R}$ with the metric $g_{\mathbf{H}}:=e^{2 t} g_{0}^{n-1}-d t^{2}$.

The other entry that requires some comment is the last row. To simplify things let $\alpha=0$ and $K_{0}=-1$ so that $\bar{g}_{w}=\cos ^{2}(t) g-d t^{2}$ where $g$ is the standard metric on the Riemannian manifold $\mathbb{R}_{0}^{n-1}(-1)$ of constant sectional curvature -1 . This metric has coordinate singularities at $t= \pm \pi / 2$ but it is isometric to an open strip in the geodesically complete anti-de Sitter space $\mathbb{R}_{1}^{n}(-1)$. It is also convenient to give this space a name:

Definition 2.7. Denote by $\left(\mathbf{S}_{1}^{n}(-1), g_{\mathbf{S}}\right)$ the space

$$
\mathbf{S}_{1}^{n}(-1):=\mathbb{R}_{0}^{n-1}(-1) \times(-\pi / 2, \pi / 2)
$$

with the metric $g_{\mathbf{S}}:=\cos ^{2}(t) g-d t^{2}$ where $g$ is the standard metric on $\mathbb{R}_{0}^{n-1}(-1)$.

Let $\gamma: \mathbb{R} \rightarrow \mathbb{R}_{1}^{n}(-1)$ be a unit speed timelike geodesic in the antide Sitter space. For each $t \in \mathbb{R}$ let $M_{t}$ be the totally geodesic hypersurface of $\mathbb{R}_{1}^{n}(-1)$ through $\gamma(t)$ and orthogonal to $\gamma^{\prime}(t)$. The set $\left\{M_{t}: t \in \mathbb{R}\right\}$ forms a smooth foliation of $\mathbb{R}_{1}^{n}(-1)$. This lets us define the time function of the geodesic $\gamma$ by

Definition 2.8. Let $\gamma: \mathbb{R} \rightarrow \mathbb{R}_{1}^{n}(-1)$ be a unit speed timelike geodesic. Then the time function defined by $\gamma$ is the function $\tau: \mathbb{R}_{1}^{n}(-1) \rightarrow \mathbb{R}$ given by

$$
\tau(x)=t \quad \text { if } x \in M_{t}
$$

\subsection{Two Dimensional Rigidity Results.}

The results in this section are lemmas that are needed to prove our higher dimensional rigidity results. 
Let $\left(M^{2}, g\right)$ be a semi-Riemannian surface. Let $\nabla$ denote the Riemannian connection on $M^{2}$. Then the curvature of $\left(M^{2}, g\right)$ is defined to be

$$
K=\langle R(X, Y) Y, X\rangle
$$

where $X, Y$ are orthonormal. Now assume that $M$ is oriented and let $e_{1}, e_{2}$ denote an oriented orthonormal frame defined locally on $M$. Let $\sigma^{1}, \sigma^{2}$ denote the dual one-forms defined in the domain of definition of the moving frame, i.e. if $I$ is the identity map on tangent spaces then $I=e_{1} \sigma^{1}+e_{2} \sigma^{2}$. Let $\omega_{i}^{j}$ denote the connection forms so that

$$
\nabla e_{i}=e_{1} \omega_{i}^{1}+e_{2} \omega_{i}^{2}
$$

By a standard calculation

$$
K d A=K \sigma^{1} \wedge \sigma^{2}=\varepsilon_{1} d \omega_{2}^{1},
$$

where $\varepsilon_{1}:=\left\langle e_{1}, e_{1}\right\rangle= \pm 1$.

Theorem 2.9. (Two Dimensional Rigidity) Let $g_{0}$ denote the standard flat metric on $\mathbb{R}_{k}^{2}$ and let $g$ be any other metric so that $g=g_{0}$ outside of some compact set $C \subset \mathbb{R}^{2}$ and the curvature $K$ of $g$ does not change sign, i.e. $K \geq 0$ everywhere or $K \leq 0$ everywhere. Then $K_{g} \equiv 0$ and $\left(\mathbb{R}^{2}, g\right)$ is isometric to $\left(\mathbb{R}_{k}^{2}, g_{0}\right)$.

Proof. Let $e_{1}^{0}, e_{2}^{0}$ be the standard basis of $\mathbb{R}^{2}$, i.e. $e_{1}^{0}=\left[\begin{array}{l}1 \\ 0\end{array}\right], e_{2}^{0}=\left[\begin{array}{l}0 \\ 1\end{array}\right]$. We claim there is a smooth orthonormal frame $e_{1}, e_{2}$ for $g$ so that $e_{i}=e_{i}^{0}$ on the complement of $C$. If $g$ is positive definite (i.e. $k=0$ ), then $e_{1}, e_{2}$ can be constructed by applying the Gram-Schmidt orthogonalization process to $e_{1}^{0}, e_{2}^{0}$ with respect to $g$. The same argument works if $g$ is negative definite $(k=2)$. This leaves the case where $k=1$, whence $g_{0}\left(e_{1}^{0}, e_{1}^{0}\right)=-1$ and $g_{0}\left(e_{2}^{0}, e_{2}^{0}\right)=+1$.

Let $O_{g}\left(\mathbb{R}^{2}\right)$ be the bundle of oriented $g$ orthonormal frames tangent to $\mathbb{R}^{2}$. That is the fiber at $P \in \mathbb{R}^{2}$ is the set of ordered pairs $\left(v_{1}, v_{2}\right)$ where

$$
\begin{gathered}
g\left(v_{1}, v_{1}\right)=-1=-g\left(v_{2}, v_{2}\right), \\
g\left(v_{1}, v_{2}\right)=0,
\end{gathered}
$$

and the orientation of $v_{1}, v_{2}$ agrees with the orientation of $e_{1}^{0}, e_{2}^{0}$. This fiber is diffeomorphic to two disjoint copies of $\mathbb{R}$. As $\mathbb{R}^{2}$ is contractible the bundle $O_{g}\left(\mathbb{R}^{2}\right)$ is trivial (cf. [22]), that is equivalent to a product bundle. A smooth 
$\mathbb{R}$ valued function defined on a neighborhood of the closure of $\mathbb{R}^{2} \backslash C$ extends to $\mathbb{R}^{2}$. Therefore, the section $e_{1}^{0}, e_{2}^{0}$ of $O_{g}\left(\mathbb{R}^{2}\right)$ can be extended from $\mathbb{R}^{2} \backslash C$ to a section $e_{1}, e_{2}$ defined on all of $\mathbb{R}^{2}$.

Now let $D$ be a bounded domain with smooth boundary so that $C \subseteq D$. Then

$$
\int_{D} K d A=\varepsilon_{1} \int_{D} d \omega_{2}^{1}=\varepsilon_{1} \int_{\partial D} \omega_{2}^{1}
$$

But $\nabla e_{i}=\nabla e_{i}^{0}=0$ on $\mathbb{R}^{2} \backslash C$ so $\omega_{2}^{1}=0$ on $\mathbb{R}^{2} \backslash C$. Thus the last equation implies $\int_{D} K d A=0$. As $K$ does not change sign this implies $K \equiv 0$. It is a standard result that $\left(\mathbb{R}^{2}, g\right)$ is isometric to $\left(\mathbb{R}_{k}^{2}, g_{0}\right)$ (cf. [40]). We note that it is also possible to use the form of the Gauss-Bonnet theorem for two dimensional space times in [5] to complete the proof in the case $k=1$.

The following variant of this will be used in the proof of Theorem 4.2.

Corollary 2.10. Let $g_{0}$ denote the standard flat metric on $\mathbb{R}_{k}^{2}$ and let $D \subset$ $\mathbb{R}_{k}^{2}$ be a connected domain with smooth boundary and compact closure in $\mathbb{R}_{k}^{2}$. Let $g$ be any other metric on $D$ so that $g=g_{0}$ in some neighborhood of $\partial D$ and the curvature $K$ of $g$ does not change sign. Then $K_{g} \equiv 0$.

We now turn to rigidity on the sphere.

Theorem 2.11. (Toponogov, [37]) Let $\left(S^{2}, g\right)$ be the two dimensional sphere with a metric so that the Gaussian curvature satisfies $1 \leq K$. Then any simple closed geodesic $\gamma$ has length at most $2 \pi$, and if the length of $\gamma$ is $2 \pi$ then $\left(S^{2}, g\right)$ is isometric to the standard sphere $\left(S^{2}, g_{0}\right)$ and $\gamma$ is a great circle.

We owe both the statement and proof of the following result to E. Calabi [6].

Theorem 2.12. Let $\left(S^{2}, g\right)$ be the two dimensional sphere with a metric of class $C^{1,1}$ whose Gaussian curvature satisfies $0 \leq K \leq 1$. Then any simple closed geodesic $\gamma$ on $\left(S^{2}, g\right)$ has length at least $2 \pi$. If the length of $\gamma$ is $2 \pi$, then either $\left(S^{2}, g\right)$ is isometric to the standard round sphere $\left(S^{2}, g_{0}\right)$ and $\gamma$ is a great circle on $\left(S^{2}, g_{0}\right)$ or $\left(S^{2}, g\right)$ is isometric to a circular cylinder of circumference $2 \pi$ capped by two unit hemispheres and $\gamma$ is a belt around the cylinder. Thus if $K$ is continuous (for example when $g$ is at least $C^{2}$ ) or if $K>0$ then $\left(S^{2}, g\right)$ is isometric to the standard round sphere.

The lower bound on the length of a simple closed closed geodesic is well known (cf. Remark 2.14), it is the rigidity result that is of interest here. 
Lemma 2.13. Let $k(t)$ be an $L^{\infty}$ function on $[0, \infty)$ so that $0 \leq k(t) \leq 1$ and let $y(t)$ be defined by the initial value problem

$$
y^{\prime \prime}(t)+k(t) y(t)=0, \quad y(0)=1, \quad y^{\prime}(0)=0 .
$$

Denote the smallest positive zero of $y$ by $\beta$ (it may be that $\beta=\infty$ ). Then $0 \leq-y^{\prime}(t) \leq 1$ for $0 \leq t \leq \beta$. If $y^{\prime}\left(t_{0}\right)=-1$ for some $t_{0} \in[0, \beta]$, then $t_{0}=\beta<\infty, \beta \geq \pi / 2$ and

$$
\begin{aligned}
& y(t)=\left\{\begin{array}{rl}
1, & 0 \leq t<\beta-\pi / 2 \\
\cos (t-(\beta-\pi / 2)), & \beta-\pi / 2<t \leq \beta
\end{array},\right. \\
& k(t)= \begin{cases}0, & 0 \leq t<\beta-\pi / 2 \\
1, & \beta-\pi / 2<t \leq \beta .\end{cases}
\end{aligned}
$$

If $k$ is continuous, for example if $y$ is $C^{2}$, and $y^{\prime}\left(t_{0}\right)=-1$ with $t_{0} \leq \beta$, then $t_{0}=\beta=\pi / 2, k \equiv 1$ and $y(t)=\cos (t)$ on $[0, \pi / 2]$.

Proof. Note on the interval $[0, \beta)$ that $\left(y^{\prime}\right)^{\prime}=y^{\prime \prime}=-k y \leq 0$ as $y>0$ and $k \geq 0$ on $[0, \beta)$. Thus $y^{\prime}$ is monotone decreasing on $[0, \beta]$. As $y^{\prime}(0)=0$ this implies $y^{\prime} \leq 0$ on $[0, \beta]$. Thus

$$
\left(y^{2}+\left(y^{\prime}\right)^{2}\right)^{\prime}=2 y y^{\prime}+2 y^{\prime} y^{\prime \prime}=2 y y^{\prime}-2 y^{\prime} k y=2 y y^{\prime}(1-k) \leq 0
$$

on $[0, \beta)$ as $k \leq 1$ and $y^{\prime} \leq 0$. Using the initial conditions for $y$ and continuity the last inequality implies

$$
y^{2}+\left(y^{\prime}\right)^{2} \leq 1 \quad \text { on } \quad[0, \beta] .
$$

These inequalities imply $0 \leq-y^{\prime} \leq 1$ on $[0, \beta]$.

If $t_{0} \in[0, \beta]$ and $y^{\prime}\left(t_{0}\right)=-1$, then the inequality (2.15) implies $y\left(t_{0}\right)=0$. But from the definition of $\beta$ as the smallest positive zero of $y$ this implies $t_{0}=\beta$. Then $y(\beta)^{2}+y^{\prime}(\beta)^{2}=1$ and equation (2.14) yields $y^{\prime}(1-k) \equiv 0$ on $[0, \beta)$. As $y^{\prime}$ is monotone decreasing on $[0, \beta)$, there is a point $t_{1} \in[0, \beta)$ so that $y^{\prime} \equiv 0$ on $\left[0, t_{1}\right]$ and $0>y^{\prime}>-1$ on $\left(t_{1}, \beta\right)$. Then on $\left[0, t_{1}\right]$ we have $y \equiv 1$ and $k \equiv 0$ (as $\left.k y=-y^{\prime \prime}=0\right)$. Also $y^{\prime}(1-k) \equiv 0$ and $y^{\prime} \neq 0$ on $\left(t_{1}, \beta\right)$ implies $k \equiv 1$ on $\left(t_{1}, \beta\right)$. But $y\left(t_{1}\right)=1$ and $y^{\prime}\left(t_{1}\right)=0$ so $y(t)=\cos \left(t-t_{1}\right)$ on $\left(t_{1}, \beta\right)$. As $y(\beta)=0$ this implies $t_{1}=\beta-\pi / 2$ on $\left(t_{1}, \beta\right)$. This completes the proof. 
Proof of the theorem. Let $c:[0, L] \rightarrow S^{2}$ be a unit speed parameterization of the closed geodesic $\gamma$, and let $\mathbf{n}$ be a unit normal along $c$. For each $s \in[0, L]$ let $\beta(s)$ be the cut distance from the curve $\gamma$ along the geodesic $t \mapsto \exp _{c(s)}(\operatorname{tn}(s))$. Define map $F(s, t)$ on the set of ordered pairs $(s, t)$ with $s \in[0, L]$ and $0 \leq t \leq \beta(s)$ by

$$
F(s, t)=\exp _{c(s)}(\operatorname{tn}(s)), \quad 0 \leq s \leq L, \quad 0 \leq t \leq \beta(s) .
$$

Then $s, t$ are Fermi coordinates on the disk $M$ bounded by $\gamma$ and with inner normal $\mathbf{n}$. In these coordinates the metric $g$, Gaussian curvature $K$ and the area form $d A$ are given by

$$
g=E^{2} d s^{2}+d t^{2}, \quad K=\frac{-E_{t t}}{E}, \quad d A=E d s d t .
$$

And because $c$ is a geodesic $E(s, 0) \equiv 1$ and $E_{t}(s, 0) \equiv 0$. Thus for fixed $s$ the function $y(t):=E(s, t)$ satisfies $y^{\prime \prime}+K y=0, y(0)=1$, and $y^{\prime}(0)=0$ as in the lemma.

Now apply the Gauss-Bonnet theorem to the disk $M$. As the boundary is a geodesic, the boundary term of the formula drops out:

$$
\begin{array}{rlrl}
2 \pi & =\int_{M} K d A=\int_{0}^{L} \int_{0}^{\beta(s)}-E_{t t} d t d s \\
& =\int_{0}^{L}\left(-E_{t}(s, \beta(s))\right) d s \quad\left(\text { as } E_{t}(s, 0)=0\right) \\
& \leq \int_{0}^{L} 1 d s & \\
& =L . &
\end{array}
$$

which proves the required lower bound on the length of $\gamma$. If $L=2 \pi$, then $E_{t}(s, \beta(s))=-1$ for all $s \in[0, L]$. Again by the lemma in the coordinates $s, t$ on $M$

$$
K(s, t)= \begin{cases}0, & 0 \leq t<\beta(s)-\pi / 2, \\ 1, & \beta(s)-\pi / 2<t \leq \beta(s) .\end{cases}
$$

Let $M_{+1}$ denote the interior of the set $\{x \in M: K(x)=+1\}$ so that

$$
M_{+1}=\left\{\exp _{c(s)}(\operatorname{tn}(s)): s \in[0,2 \pi], \beta(s)-\pi / 2<t \leq \beta(s)\right\} .
$$

Let $s_{0} \in[0,2 \pi]$ be a point where $\beta(s)$ is maximal. Then the open disk $B\left(x_{0}, \pi / 2\right)$ of radius $\pi / 2$ about $x_{0}:=\exp _{c\left(s_{0}\right)}\left(\beta\left(s_{0}\right) \mathbf{n}\left(s_{0}\right)\right)$ is contained in 
$M_{+1}$, for if not it would meet $\partial M_{+1}$ at some point $\exp _{c(s)}((\beta(s)-\pi / 2) \mathbf{n}(s))$ and this point is a distance of $\beta(s)-\pi / 2$ from $\gamma$. Thus the distance of $x_{0}=\exp _{c\left(s_{0}\right)}\left(\beta\left(s_{0}\right) \mathbf{n}\left(s_{0}\right)\right)$ to $\gamma$ is less than $\pi / 2+(\beta(s)-\pi / 2)=\beta(s)$, which contradicts the maximality of $\beta\left(s_{0}\right)$. Thus $B\left(x_{0}, \pi / 2\right) \subseteq M_{+1}$. But using the Gauss-Bonnet theorem and $K \equiv+1$ on $M_{+1}$

$$
2 \pi \geq \int_{M_{+1}} K d A=\operatorname{Area}\left(M_{+1}\right) \geq \operatorname{Area}\left(B\left(x_{0}, \pi / 2\right)\right)=2 \pi .
$$

So $M_{+1}=B\left(x_{0}, \pi / 2\right)$. From this it follows $s \mapsto \beta(s)$ is constant and thus the disk $M$ bounded by $\gamma$ and with inner normal $\mathbf{n}$ is a cylinder of circumference $2 \pi$ capped at one end with a hemisphere. The same argument applied to the disk bounded by $\gamma$ and having $-\mathbf{n}$ as inward normal shows $\left(S^{2}, g\right)$ is two of these capped cylinders glued together along $\gamma$, which is equivalent to the statement of the theorem.

Remark 2.14. If one is only interested in the length of closed geodesics, there are higher dimensional versions of Theorem 2.12. If $(M, g)$ is a compact orientable Riemannian manifold of even dimension with sectional curvatures satisfying, $0<K_{M} \leq 1$, then Klingenberg has shown every closed geodesic has length $\geq 2 \pi$. This is equivalent to his well known lower bound on the injectivity radius of compact oriented even dimensional manifolds. For a proof see the book [7, Chapter 5]. For odd dimensional manifolds another theorem of Klingenberg's implies if $(M, g)$ is a compact simply connected manifold of whose sectional curvature satisfies $1 / 4<K_{M} \leq 1$, then any closed geodesic of $(M, g)$ has length at least $2 \pi$. Again a proof can be found in [7, Chapter 5]. (The original proofs of Klingenberg are in [25, 26].) We also note that in dimension 3 for any $\varepsilon>0$ there are examples of metrics $g$ on $M=S^{3}$ (due to Berger) so that the sectional curvatures satisfy $1 / 9-\varepsilon \leq K_{M} \leq 1$, but $\left(S^{3}, g\right)=(M, g)$ has a geodesic of length less than $2 \pi$. Cf. [7, Example 3.35, page 70]. To the best of our knowledge there is no known rigidity result in these theorems.

\section{Basic Comparison Results.}

Most of the results in this section will be stated as results about systems of ordinary differential equations (usually in the form of solutions to matrix valued differential equations). These results then apply to the second fundamental forms (or Weingarten maps) of parallel families of hypersurfaces in a Semi-Riemannian manifold. 


\subsection{Comparisons for Parallel Hypersurfaces.}

In this section we will prove a comparison theorem for solutions of the matrix Riccati equation.

Let $E$ be an $n$ dimensional real inner product space with an inner product of index $k$. The inner product will be denoted by $\langle$,$\rangle . A linear map A$ : $E \rightarrow E$ is self-adjoint if $\langle A x, y\rangle=\langle x, A y\rangle$ for all $x, y \in E$. Unlike the case of positive definite spaces a self-adjoint linear map need not have real eigenvalues. (An easy example of this is $E=\mathbb{R}^{2},\langle\rangle=,d x^{2}-d y^{2}$ and $A=\left[\begin{array}{cc}0 & 1 \\ -1 & 0\end{array}\right]$. Then the eigenvalues of $A$ are $\pm \sqrt{-1}$.)

A self-adjoint linear map $A$ is positive definite if and only if $\langle A x, x\rangle>0$ for all $x \neq 0$. The eigenvalues of a positive definite linear map are real. (This can be seen by using the principal axis theorem to diagonalize the inner product $\langle$,$\rangle with respect to the positive definite inner product \langle A$,$\rangle .)$ However the eigenvalues of a positive definite map need not be positive. In fact if the index of the inner product is $k$ then a positive definite map will have exactly $k$ negative eigenvalues, however having $k$ negative eigenvalues is not enough to insure $A$ is positive definite. Unlike the positive definite case the identity map is not positive definite. A linear map is positive semidefinite iff $\langle A x, x\rangle \geq 0$ for all $x \in E$. If $A$ and $B$ are self-adjoint, then we write $A \leq B$ iff $B-A$ is positive semi-definite, and $A<B$ iff $B-A$ is positive definite. The set of positive definite maps forms a cone.

Lemma 3.1. If $A \geq 0$ and $\left\langle A x_{0}, x_{0}\right\rangle=0$, then $A x_{0}=0$.

Proof. Because $A$ is positive semi-definite and $\left\langle A x_{0}, x_{0}\right\rangle=0$ we have for all $h \in E$ that $0 \leq\left\langle A\left(x_{0}+h\right),\left(x_{0}+h\right)\right\rangle=2\left\langle A x_{0}, h\right\rangle+\langle A h, h\rangle$ This can only be non-negative for all $h$ if $\left\langle A x_{0}, h\right\rangle=0$ for all $h$. This implies $A x_{0}=0$.

For $i=1,2$ let $t \mapsto R_{i}(t)$ be smooth maps from the real numbers $\mathbb{R}$ to the space of self-adjoint linear maps on $E$. Also let $A_{1}$ and $A_{2}$ be self-adjoint linear maps on $E$ and denote by $I$ the identity operator on $E$. Define two maps $t \mapsto F_{i}(t)$ from the reals to the space of linear maps on $E$ by the initial value problems,

$$
F_{i}^{\prime \prime}(t)+R_{i}(t) F_{i}(t)=0, \quad F_{i}(0)=I, \quad F_{i}^{\prime}(0)=-A_{i} ; \quad i=1,2 .
$$

At the points $t$ where $F_{i}(t)$ is invertible set

$$
S_{i}(t)=-F_{i}^{\prime}(t) F_{i}(t)^{-1} ; \quad i=1,2 .
$$


Let $F_{i}(t)^{*}$ be the transpose of $F_{i}(t)$ with respect to the inner product $\langle$, (defined by $\left\langle F_{i}(t)^{*} x, y\right\rangle=\left\langle x, F_{i}(t) y\right\rangle$ ). Then using the equation (3.1) it follows

$$
\frac{d}{d t}\left(F_{i}(t)^{*} F_{i}^{\prime}(t)-F_{i}^{\prime}(t)^{*} F(t)\right)=0 ; \quad i=1,2 .
$$

which implies the linear maps $S_{i}(t)$ are self-adjoint at all points where they are defined. It also follows directly from (3.1) that in some neighborhood of 0 , the $S_{i}(t)$ satisfy the initial value problem

$$
S_{i}^{\prime}(t)=S_{i}(t)^{2}+R_{i}(t), \quad S_{i}(0)=A_{i} ; \quad i=1,2 .
$$

Theorem 3.2. With the notation above, if both $S_{1}(t)$ and $S_{2}(t)$ are defined on all of the interval $[0, b]$ and if

$$
A_{1} \leq A_{2}, \quad R_{1}(t) \leq R_{2}(t) \text { for all } t \in[0, b],
$$

then $S_{1}(t) \leq S_{2}(t)$ on $[0, b]$. If $S_{1}(b)=S_{2}(b)$, then $A_{1}=A_{2}$ and $R_{1}(t) \equiv R_{2}(t)$ on $[0, b]$.

Proof. We first prove this under the assumption $A_{1}<A_{2}$ and $R_{1}(t)<R_{2}(t)$ for all $t \in[0, b]$ and show in this case $S_{1}(t)<S_{2}(t)$ for $t \in[0, b]$. Assume, toward a contradiction, this is false. Then there is a smallest $t_{0}$ so that $S_{2}\left(t_{0}\right)-S_{1}\left(t_{0}\right)$ is not positive definite. By continuity $S_{2}\left(t_{0}\right)-S_{1}\left(t_{0}\right)$ is positive semi-definite. As it is not positive definite there is a nonzero vector $x_{0} \in E$ so that $\left\langle\left(S_{2}\left(t_{0}\right)-S_{1}\left(t_{0}\right)\right) x_{0}, x_{0}\right\rangle=0$. By Lemma 3.1 this yields $S_{1}\left(t_{0}\right) x_{0}=S_{2}\left(t_{0}\right) x_{0}$.

Let $f(t):=\left\langle\left(S_{2}(t)-S_{1}(t)\right) x_{0}, x_{0}\right\rangle$. Then, using $S_{1}\left(t_{0}\right) x_{0}=S_{2}\left(t_{0}\right) x_{0}$,

$$
\begin{aligned}
f^{\prime}\left(t_{0}\right)= & \left\langle\left(S_{2}^{\prime}\left(t_{0}\right)-S_{1}^{\prime}\left(t_{0}\right)\right) x_{0}, x_{0}\right\rangle \\
= & \left\langle S_{2}\left(t_{0}\right) x_{0}, S_{2}\left(t_{0}\right) x_{0}\right\rangle-\left\langle S_{1}\left(t_{0}\right) x_{0}, S_{1}\left(t_{0}\right) x_{0}\right\rangle \\
& \quad+\left\langle\left(R_{2}\left(t_{0}\right)-R_{1}\left(t_{0}\right)\right) x_{0}, x_{0}\right\rangle \\
= & \left\langle\left(R_{2}\left(t_{0}\right)-R_{1}\left(t_{0}\right)\right) x_{0}, x_{0}\right\rangle \\
> & 0 .
\end{aligned}
$$

But $f$ is positive on $\left[0, t_{0}\right)$ and $f\left(t_{0}\right)=0$ thus $f^{\prime}\left(t_{0}\right) \leq 0$. This contradicts the last equation and completes the proof in the case $A_{2}-A_{1}$ and $R_{2}(t)-R_{1}(t)$ are positive definite.

For the general case let $B$ be any positive definite matrix. Then for small $\delta>0$ define $S_{\delta}$ by the initial value problem

$$
S_{\delta}^{\prime}(t)=S_{\delta}(t)^{2}+R_{2}(t)+\delta B, \quad S_{\delta}(0)=A_{2}+\delta B .
$$


By what has already been done we have $S_{1}(t)<S_{\delta}(t)$ on $[0, b]$. Using that $S_{\delta}$ depends continuously on $\delta$,

$$
S_{2}(t)=\lim _{\delta \downarrow 0} S_{\delta}(t) \geq S_{1}(t) .
$$

This proves the inequality in the general case.

Assume $S_{1}(b)=S_{2}(b)$ and let $t_{0} \in[0, b)$. Then define $S$ on $\left[t_{0}, b\right]$ by

$$
S^{\prime}(t)=S(t)^{2}+R_{1}(t), \quad S\left(t_{0}\right)=S_{2}\left(t_{0}\right)
$$

so $S$ satisfies the same differential equation as $S_{1}$ and agrees with $S_{2}$ at $t_{0}$. By what has already been shown this implies $S_{1}(t) \leq S(t) \leq S_{2}(t)$ on $\left[t_{0}, b\right]$. As $S_{1}(b)=S_{2}(b)$ this yields $S(b)=S_{1}(b)$. But $S_{1}$ and $S$ satisfy the same first order equation and agree at the point $b$, so $S \equiv S_{1}$ on $\left[t_{0}, b\right]$. In particular $S_{1}\left(t_{0}\right)=S\left(t_{0}\right)=S_{2}\left(t_{0}\right)$. As $t_{0}$ was arbitrary $S_{1} \equiv S_{2}$. By uniqueness this implies $A_{1}=A_{2}$ and $R_{1} \equiv R_{2}$.

A standard part of the comparison theory in the positive definite case is, with the notation above, if $S_{2}(t)$ is defined on all of $[0, b]$ then so is $S_{1}(t)$. This does not hold on in the general indefinite metric case. We give two examples on $\mathbb{R}^{2}$ with the inner product $\langle\rangle=,d x^{2}-d y^{2}$. First let $A_{1}=A_{2}=0, R_{1}(t) \equiv 0$ and $R_{2}(t) \equiv\left[\begin{array}{ll}1 & 0 \\ 0 & 0\end{array}\right]$. Then $R_{1} \leq R_{2}, S_{1}=0$ is defined on all of $[0, \infty)$ but $S_{2}(t)=\left[\begin{array}{cc}\tan t & 0 \\ 0 & 0\end{array}\right]$ is only defined on $[0, \pi / 2)$. In the second example again let $A_{1}=A_{2}=0$, but let $R_{1}(t) \equiv\left[\begin{array}{ll}0 & 0 \\ 0 & 1\end{array}\right]$ and $R_{2}(t) \equiv 0$. Again $R_{1}(t) \leq R_{2}(t) . S_{1}(t)=\left[\begin{array}{cc}0 & 0 \\ 0 & \tan t\end{array}\right]$ is only defined on $[0, \pi / 2)$ but $S_{2}(t)=0$ is defined on all of $[0, \infty)$.

However if there is a two sided curvature bound, then there is a an estimate of the size of the domain of definition.

Proposition 3.3. Let $R_{1}(t), R_{2}(t), R_{3}(t)$ be continuous functions of $t \in \mathbb{R}$ with values in the symmetric linear maps on $(E,\langle\rangle$,$) so that R_{1} \leq R_{2} \leq R_{3}$ on $[0, b]$. For $i=1,2,3$, define $S_{i}(t)$ by the initial value problems $S_{i}^{\prime}=S_{i}^{2}+R_{i}$ and assume $S_{1}(0) \leq S_{2}(0) \leq S_{3}(0)$. If $S_{1}$ and $S_{3}$ are defined on all of $[0, b)$, then so is $S_{2}$.

Proof. Let $\left[0, t_{0}\right)$ be the maximal interval of definition of $S_{2}$. If $t_{0} \geq b$ we are done, so assume, toward a contradiction, $t_{0}<b$. By the comparison theorem $S_{1}(t) \leq S_{2}(t) \leq S_{3}(t)$ on $\left[0, t_{0}\right)$. As $S_{1}$ and $S_{3}$ are continuous on $\left[0, t_{0}\right]$ their ranges are bounded. Thus there are self-adjoint $A$ and $B$ so that $A \leq S_{1}(t) \leq S_{3}(t) \leq B$. But the set $\mathcal{C}=\{C: A \leq C \leq B\}$ is a 
compact subset of the space of self-adjoint linear maps and $S_{2}(t) \in \mathcal{C}$ for $t \in\left[0, t_{0}\right)$. Therefore there is a sequence $t_{k} \nearrow t_{0}$ so that $\lim _{k \rightarrow \infty} S_{2}\left(t_{k}\right)$ exists, say $\lim _{k \rightarrow \infty} S_{2}\left(t_{k}\right)=G$. Choose any norm $\|\cdot\|$ on the space of self-adjoint linear maps on $E$. Then, as $S_{2}(t)$ stays in a compact set, there is a constant $c_{0}$ so that $\left\|S_{2}(t)^{2}+R_{2}(t)\right\| \leq c_{0}$ for all $t \in\left[0, t_{0}\right)$. Thus the differential equation for $S_{2}$ implies $\left\|S_{2}(t)-S_{2}(s)\right\| \leq c_{0}|s-t|$ for all $t, s \in\left[0, t_{0}\right)$. Then $\lim _{t} / t_{0} S(t)=\lim _{k \rightarrow \infty} S_{2}\left(t_{k}\right)=G$. Define $S(t)$ by $S^{\prime}=S^{2}+R_{2}$ and $S\left(t_{0}\right)=G$. Then by uniqueness of solutions to initial value problems we find that $S_{2}=S$ in a neighborhood of $t_{0}$, contradicting that $\left[0, t_{0}\right)$ was the maximal interval of definition of $S_{2}$

\subsubsection{Comparisons for Curvature Tensors.}

For the later applications we need not just the above comparison theorems for the Weingarten map, but also comparison theorems for the intrinsic curvature tensor. The idea is to use the Gauss curvature equation to relate the estimates on Weingarten map to the sectional curvature. We start by giving some linear algebraic results.

Recall the inner product $\langle$,$\rangle induces an inner product (also denoted$ by $\langle\rangle$,$) on \bigwedge^{2} E$. On decomposable elements it is given by,

$$
\left\langle x_{1} \wedge x_{2}, y_{1} \wedge y_{2}\right\rangle=\left\langle x_{1}, y_{1}\right\rangle\left\langle x_{2}, y_{2}\right\rangle-\left\langle x_{1}, y_{2}\right\rangle\left\langle x_{2}, y_{1}\right\rangle .
$$

Also a linear map $A: E \rightarrow E$ induces a linear map $\bigwedge^{2}(A): \bigwedge^{2} E \rightarrow \bigwedge^{2} E$ given on decomposable elements by $\wedge^{2}(A) x \wedge y=A x \wedge A y$.

Definition 3.4. If $A, B$ are self-adjoint linear maps on $E$ then define $\bigwedge^{2}(A) \preceq \bigwedge^{2}(B)$ to mean that $\left\langle\bigwedge^{2}(A) x \wedge y, x \wedge y\right\rangle \leq\left\langle\bigwedge^{2}(B) x \wedge y, x \wedge y\right\rangle$ for all decomposable $x \wedge y$. This is equivalent to

$$
\langle A x, x\rangle\langle A y, y\rangle-\langle A x, y\rangle^{2} \leq\langle B x, x\rangle\langle B y, y\rangle-\langle B x, y\rangle^{2}
$$

holding for all $x, y \in E$.

Lemma 3.5. If $A$ is self-adjoint and $\left\langle\bigwedge^{2}(A) x \wedge y, x \wedge y\right\rangle=0$ for all decomposable vectors $x \wedge y$, then $A$ has rank one and so $\bigwedge^{2}(A)=0$. If $A$ is self-adjoint and $0 \leq A$ (or $A \leq 0$ ) then $\bigwedge^{2}(A) \succeq 0$.

Proof. If $\left\langle\bigwedge^{2}(A) x \wedge y, x \wedge y\right\rangle=0$ then

$$
\langle A x, x\rangle\langle A y, y\rangle-\langle A x, y\rangle^{2}=0 .
$$


Let $h \in E$. Then replace $x$ by $x+t h$ in the last equation and take $\left.\frac{d}{d t}\right|_{t=0}$ to get

$$
2\langle A x, h\rangle\langle A y, y\rangle-2\langle A x, y\rangle\langle h, A y\rangle=0
$$

for all $x, y, h \in E$. If $A=0$ there in nothing to prove. Thus assume $A \neq 0$. Then, as $A$ is self-adjoint, there is a $y \in E$ so that $\langle A y, y\rangle \neq 0$. From the last equation we see if $\langle h, A y\rangle=0$, then also $\langle A x, h\rangle=0$. This implies $A x$ is a scalar multiple of $A y$. As $x$ was arbitrary, this shows $A y$ spans the range of $A$, so $A$ has rank one.

If $A \geq 0$ (or $A \leq 0$ ), then the Cauchy-Schwartz inequality applied to the inner product $\langle A \cdot, \cdot\rangle$ implies $\langle A x, x\rangle\langle A y, y\rangle-\langle A x, y\rangle^{2} \geq 0$, i.e. $\bigwedge^{2}(A) \succeq 0$.

Lemma 3.6. If $A, B$ are self-adjoint and $0 \leq A \leq B$ (or $B \leq A \leq 0$ ), then $\bigwedge^{2}(A) \preceq \bigwedge^{2}(B)$. If also either $A$ or $B$ is positive definite and $\left\langle\bigwedge^{2}(A) x \wedge\right.$ $y, x \wedge y\rangle=\left\langle\Lambda^{2}(B) x \wedge y, x \wedge y\right\rangle$ for all $x, y \in E$ then $A=B$.

Proof. We first assume $B$ is positive definite. Let $x, y \in E$ be linearly independent and let $V=\operatorname{Span}(x, y)$. Let $\alpha$ and $\beta$ be the inner products defined on $V$ by $\alpha(u, v)=\langle A u, v\rangle, \beta(u, v)=\langle B u, v\rangle$. As $B$ is positive definite so is $\beta$. Thus by the principal axis theorem the forms $\alpha$ and $\beta$ can be simultaneously diagonalized. Therefore there is a basis $x_{1}, y_{1}$ of $V$ with $x_{1} \wedge y_{1}=x \wedge y$ and $\left\langle A x_{1}, y_{1}\right\rangle=\left\langle B x_{1}, y_{1}\right\rangle=0$. But for all $u \in E$, $0 \leq\langle A u, u\rangle \leq\langle B u, u\rangle$ so

$$
\begin{aligned}
\left\langle\bigwedge^{2}(A) x \wedge y, x \wedge y\right\rangle & =\left\langle A x_{1}, x_{1}\right\rangle\left\langle A y_{1}, y_{1}\right\rangle \\
& \leq\left\langle B x_{1}, x_{1}\right\rangle\left\langle B y_{1}, y_{1}\right\rangle=\left\langle\bigwedge^{2}(B) x \wedge y, x \wedge y\right\rangle .
\end{aligned}
$$

If equality holds then $\left\langle A x_{1}, x_{1}\right\rangle=\left\langle B x_{1}, x_{1}\right\rangle$ and $\left\langle A y_{1}, y_{1}\right\rangle=\left\langle B y_{1}, y_{1}\right\rangle$. As $x_{1}, y_{1}$ diagonalizes both $\alpha$ and $\beta$ this implies $\langle A u, u\rangle=\langle B u, u\rangle$ for all $u \in V$. So if $\left\langle\bigwedge^{2}(A) x \wedge y, x \wedge y\right\rangle=\left\langle\bigwedge^{2}(B) x \wedge y, x \wedge y\right\rangle$ for all $x, y \in E$, then $\langle A u, u\rangle=\langle B u, u\rangle$ for all $u \in E$, which implies $A=B$.

A similar argument works if $A$ is positive definite. Finally if $B$ is only positive semi-definite then let $C$ be positive definite and let $B_{\ell}=B+\frac{1}{\ell} C$. Then $B_{\ell}$ is positive definite and $\lim _{\ell \rightarrow \infty} B_{\ell}=B$. But $0 \leq A \leq B_{\ell}$ implies $\bigwedge^{2}(A) \leq \bigwedge^{2}\left(B_{\ell}\right)$ as $B_{\ell}$ is positive definite, so a limit argument shows this also holds when $B=\lim _{\ell \rightarrow \infty} B_{\ell}$ is positive semi-definite.

Lemma 3.7. A rank one self-adjoint linear map $S: E \rightarrow E$ is of one of the two forms $S x=\langle x, e\rangle$ e or $S x=-\langle x, e\rangle$, for some $e \in E$. 
Proof. A rank one linear map is of the form $S x=\langle x, u\rangle v$ with both $u$, $v$ non-zero. The map $S$ is self-adjoint iff $\langle S x, y\rangle=\langle x, S y\rangle$, which in our case this reduces to $\langle x, u\rangle\langle y, v\rangle=\langle x, v\rangle\langle y, u\rangle$. This implies $u$ and $v$ are linearly dependent. As they are both non-zero $v=\lambda u$ for some non-zero real number $\lambda$. If $\lambda>0$ let $e=\sqrt{\lambda} u$. Then $S x=\langle x, u\rangle v=\langle x, e\rangle e$. If $\lambda<0$ set $e=-\sqrt{|\lambda|} u$. Then $S x=\langle x, u\rangle v=-\langle x, e\rangle$.

Theorem 3.8. Let $t \mapsto S(t)$ be a smooth map from $[0, b]$ to the self-adjoint maps on $E$. Assume $S$ satisfies

$$
S^{\prime}(t)=S(t)^{2}+R(t), \quad S(0)=0,
$$

where $R(t) \geq 0$ on $[0, b]$ (or $R(t) \leq 0$ on $[0, b])$. Then $\bigwedge^{2}(S(t)) \succeq 0$ on $[0, b]$. If $\left\langle\bigwedge^{2}(S(b)) x \wedge y, x \wedge y\right\rangle=0$ for all $x, y$ then there is a self-adjoint rank one map $P$ and smooth functions $u, r:[0, r] \rightarrow \mathbb{R}$ so that

$$
S(t)=u(t) P, \quad R(t)=r(t) P, \quad \text { and } \quad u^{\prime}(t)=u^{2}(t)+r(t) .
$$

Proof. We deal with the case $R(t) \geq 0$ on $[0, b]$, the case with $R(t) \leq 0$ being similar. By the comparison Theorem $3.2 S(t) \geq 0$ on $[0, b]$. By Lemma 3.5 this implies $\bigwedge^{2}(S(t)) \succeq 0$. Now assume $\left\langle\bigwedge^{2}(S(b)) x \wedge y, x \wedge y\right\rangle=0$ for all $x$, $y$. Then again by Lemma $3.5, S(b)$ has rank one or less. If $S(b)=0$ then Theorem 3.2 implies $S(t) \equiv 0$ and $R(t) \equiv 0$.

Now assume $S(b)$ has rank one. Again by Theorem 3.2 there is a $t_{0} \in[0, b)$ so that $S(t)=0$ on $\left[0, t_{0}\right]$ and $S(t)$ has rank one on $\left(t_{0}, b\right]$. By Lemma 3.7 each $t \in\left(t_{0}, b\right]$ there is $e(t) \in E$ so that $S(t)=$ $\pm\langle\cdot, e(t)\rangle e(t)$. Then $S^{\prime}(t)= \pm\left(\left\langle\cdot, e^{\prime}(t)\right\rangle e(t)+\langle\cdot, e(t)\rangle e^{\prime}(t)\right)$, and $S(t)^{2}=$ $\langle\cdot, e(t)\rangle\langle e(t), e(t)\rangle e(t)$. From the differential equation for $S$,

$$
\begin{aligned}
R(t)=S^{\prime}(t) & -S(t)^{2} \\
= & \pm\left(\left\langle\cdot, e^{\prime}(t)\right\rangle e(t)+\langle\cdot, e(t)\rangle e^{\prime}(t)\right)-\langle\cdot, e(t)\rangle\langle e(t), e(t)\rangle e(t) .
\end{aligned}
$$

Using $R(t) \geq 0$,

$$
\langle R(t) x, x\rangle= \pm 2\langle x, e(t)\rangle\left\langle x, e^{\prime}(t)\right\rangle-\langle x, e(t)\rangle^{2}\langle e(t), e(t)\rangle \geq 0
$$

for all $x$.

Claim. For any $t \in\left(t_{0}, b\right]$ the vectors $e(t)$ and $e^{\prime}(t)$ are linearly dependent. 
To see this assume for some $t$ that $e(t)$ and $e^{\prime}(t)$ are linearly independent. Then there are vectors $x_{0}, h \in E$ so that $\left\langle x_{0}, e(t)\right\rangle=1,\left\langle x_{0}, e^{\prime}(t)\right\rangle=0$, $\langle h, e(t)\rangle=0$, and $\left\langle h, e^{\prime}(t)\right\rangle=1$. Let $\lambda$ be any real number and let $x=$ $x_{0}+\lambda h$. Using this $x$ in the expression above for $\langle R(t) x, x\rangle$ implies that for all $\lambda$

$$
\langle R(t) x, x\rangle= \pm 2 \lambda-\langle e(t), e(t)\rangle \geq 0 .
$$

which is impossible. This proves the claim.

As $e(t)$ and $e^{\prime}(t)$ are linearly dependent for all $t$ the span of $e(t)$ stays constant. Thus there is a constant vector $e_{0}$ so that $\operatorname{Span} e(t)=\operatorname{Span} e_{0}$ for all $t$. Let $P$ be a non-zero rank one self-adjoint map with the range of $P$ being the span of $e_{0}$. (Say $P=\left\langle\cdot, e_{0}\right\rangle e_{0}$.) Then $S(t)=u(t) P$ for some smooth function $u$. From the equation for $S(t)$ we have $R(t)=S^{\prime}(t)-S(t)^{2}=$ $\left(u^{\prime}(t)-u(t)^{2}\right) P$ so setting $r(t)=u^{\prime}(t)-u(t)^{2}$ completes the proof.

Theorem 3.9. Let $r(t)$ be a smooth function on $[0, b]$ and a a real number. Assume there is a function $u$ on $[0, b]$ so that

$$
u^{\prime}(t)=u^{2}(t)+r(t), \quad u(0)=a .
$$

Let $A$ be a positive definite self-adjoint map on $E$, and $t \mapsto S(t)$ a map from $[0, b]$ to the self-adjoint maps on $E$ so that

$$
S^{\prime}(t)=S(t)^{2}+R(t), \quad S(0)=a A
$$

and $R$ satisfies one of the following conditions:

(1) $R(t) \geq r(t) A$ on $[0, b]$ and $u(b)>0$, or

(2) $R(t) \leq r(t) A$ on $[0, b]$ and $u(b)<0$.

Then $\bigwedge^{2}(S(b)) \succeq u(b)^{2} \bigwedge^{2}(A)$. If

$$
\left\langle\bigwedge^{2}(S(b)) x \wedge y, x \wedge y\right\rangle=u(b)^{2}\left\langle\bigwedge^{2}(A) x \wedge y, x \wedge y\right\rangle \text { for all } x, y \in E
$$

then $S(t) \equiv u(t) A$ and $R(t) \equiv r(t) A$.

Proof. We prove this under the first assumption, the proof in the second case being almost identical. By Theorem 3.2 we have $0 \leq u(b) A \leq S(b)$. By Lemma 3.6 this implies $\bigwedge^{2}(S(b)) \succeq u(b)^{2} \bigwedge^{2}(A)$. Also by Lemma 3.6 if $\left\langle\bigwedge^{2}(S(b)) x \wedge y, x \wedge y\right\rangle=u(b)^{2}\left\langle\bigwedge^{2}(\bar{A}) x \wedge y, x \wedge y\right\rangle$ for all $x, y \in E$ then $S(t) \equiv u(t) A$ and $R(t) \equiv r(t) A$. Then the uniqueness part of Theorem 3.2 implies $S(t)=u(t) A$ and $R(t)=r(t) A$. 
Theorem 3.10. Let $r(t)$ be a smooth function on $[0, b]$ and $a>0$ a real number. Assume there is a function $u$ on $[0, b]$ so that

$$
u^{\prime}(t)=u^{2}(t)+r(t), \quad u(0)=a .
$$

Let $A$ be a positive definite self-adjoint map on $E$, and $t \mapsto S(t)$ a map from $[0, b]$ to the self-adjoint maps on $E$ so that

$$
S^{\prime}(t)=S(t)^{2}+R(t), \quad S(0)=a A
$$

and assume

$$
R(t) \leq r(t) A, \text { and } S(t)>0 \text { on }[0, b] .
$$

Then $\bigwedge^{2}(S(b)) \leq u(b)^{2} \bigwedge^{2}(A)$. If

$$
\left\langle\bigwedge^{2}(S(b)) x \wedge y, x \wedge y\right\rangle=u(b)^{2}\left\langle\bigwedge^{2}(A) x \wedge y, x \wedge y\right\rangle \text { for all } x, y \in E
$$

then $S(t) \equiv u(t) A$ and $R(t) \equiv r(t) A$.

Proof. A variant on the proof of the last theorem.

Remark 3.11. In the Riemannian case there are volume comparison theorems for the volume of geodesic balls and tubes. In the Lorentzian case there are volume comparison for hypersurfaces parallel to a spacelike hypersurface. In the notation of this section these reduce to giving inequalities between $\operatorname{det}\left(F_{1}(t)\right)$ and $\operatorname{det}\left(F_{2}(t)\right)$. We now give examples to show there are no such inequalities in the general indefinite metric case. We will work on $\mathbb{R}^{2}$ with the metric $\langle\rangle=,d x^{2}-d y^{2}$. First let $A_{1}=A_{2}=0, R_{1}(t) \equiv 0$, and $R_{2}(t) \equiv\left[\begin{array}{ll}1 & 0 \\ 0 & 0\end{array}\right]$. Then $R_{1} \leq R_{2}$. Define $F_{i}$ by $F_{i}^{\prime \prime}+R_{i} F_{i}=0, F_{i}(0)=I$, $F_{i}^{\prime}(0)=A_{i}=0$. Then $F_{1}(t)=\left[\begin{array}{ll}1 & 0 \\ 0 & 1\end{array}\right]$ and $F_{2}(t)=\left[\begin{array}{cc}\cos t & 0 \\ 0 & 1\end{array}\right]$. So in this case

$$
\operatorname{det}\left(F_{1}(t)\right)=1>\cos t=\operatorname{det}\left(F_{2}(t)\right) \text { for } 0<t<2 \pi .
$$

For the second example again use $A_{1}=A_{2}=0$, but this time let $R_{1}(t) \equiv\left[\begin{array}{ll}0 & 0 \\ 0 & 1\end{array}\right]$ and $R_{2}(t) \equiv 0$. Then $R_{1}(t) \leq R_{2}(t), F_{1}=\left[\begin{array}{cc}1 & 0 \\ 0 & \cos t\end{array}\right]$, and $F_{2}(t)=\left[\begin{array}{ll}1 & 0 \\ 0 & 1\end{array}\right]$. So this time

$$
\operatorname{det}\left(F_{1}(t)\right)=\cos t<1=\operatorname{det}\left(F_{2}(t)\right)
$$

and the inequality goes in the other direction.

If $\langle$,$\rangle is positive definite and A, B$ are self-adjoint with $A \leq B$, then $\operatorname{trace}(A) \leq \operatorname{trace}(B)$. However if $\langle$,$\rangle is indefinite then A \leq B$ does not imply any inequality between $\operatorname{trace}(A)$ and $\operatorname{trace}(B)$. This is exactly where the proof of the volume comparison theorem breaks down in the indefinite metric case. 


\subsection{Comparisons for Tubes.}

Theorem 3.2 implies a comparison theorem for the second fundamental form of a hypersurface parallel to a given hypersurface. In the Riemannain case there are comparison results for tubes about submanifolds of higher codimension which have interesting applications (cf. [19]). In the semi-Riemannain case there is also a comparison result for the second fundamential forms of tubes about non-degenerate submanifolds of $(\bar{M}, \bar{g})$, but there do not seem to be applications of this result that have the same interest as the Riemannian theorem. Thus for the sake of completeness we include the statement of the result, but omit the proof. As with the results above we state this as a theorem about systems of ordinary differential equations.

Let $T$ be a subspace of $E$ and assume that the restriction of the inner product $\langle$,$\rangle to T$ is nondegenerate. Let $T^{\perp}$ be the orthogonal complement of $T$ in $E$. Then $E=T \oplus T^{\perp}$. Let $P: E \rightarrow T$ and $P^{\perp}: E \rightarrow T^{\perp}$ be the orthogonal projections. This includes the case when $T=\{0\}$, so $P^{\perp}=I$.

Theorem 3.12. For $i=1,2$, let $A_{i}: T \rightarrow T$ be a self-adjoint linear maps and $R_{i}(t)$ as in Theorem 3.2. Then define $F_{i}(t)$ by

$$
F_{i}^{\prime \prime}(t)+R_{i}(t) F_{i}(t)=0, \quad F_{i}(0)=P, \quad F_{i}^{\prime}(0)=-A_{i} P+P^{\perp} ; \quad i=1,2 .
$$

At the points where $F_{i}(t)$ is non-singular define $S_{i}(t)=-F_{i}^{\prime}(t) F_{i}(t)^{-1}$. As before the maps $S_{i}(t)$ are self-adjoint at the points where they are defined. If $S_{1}(t)$ and $S_{2}(t)$ are defined on $(0, b]$ and

$$
A_{1} \leq A_{2}, \quad R_{1}(t) \leq R_{2}(t) \quad \text { for all } t \in(0, b],
$$

then $S_{1}(t) \leq S_{2}(t)$. If $S_{1}(b)=S_{2}(b)$ then $A_{1}=A_{2}$ and $S_{1} \equiv S_{2}, R_{1} \equiv R_{2}$ on $(0, b]$.

Remark 3.13. There is one case where this is of interest, and that is when $M$ is a point $p$ in a Lorentzian manifold. Let $S_{-}(p):=\left\{u \in T(\bar{M})_{p}\right.$ : $\bar{g}(u, u)=-1\}$. Then $S_{-}(p)$ is a Riemannian manifold isometric to two disjoint copies of the hyperbolic space $\mathbb{R}_{0}^{n-1}(-1)$. Define a map $f_{r}: S_{-}(p) \rightarrow$ $\bar{M}$ by $f_{r}(u)=\exp _{p}(r u)$. If $(\bar{M}, \bar{g})$ is timelike geodesically complete and the curvature satisfies $\langle\bar{R}(X, Y) Y, X\rangle \leq 0$ for all pairs of vectors $X, Y$ spanning a timelike two plane, then for any $X$ tangent to $S_{-}(p)$ at $u$

$$
\left\langle f_{r * u} X, f_{r * u} X\right\rangle \geq \frac{1}{r^{2}}\langle X, X\rangle .
$$


This result is due to Flaherty [11] where he used it to prove a version of the Cartan-Hadamard theorem for Lorentzian manifolds. We note that this also follows from the result above as the derivative of $f_{r}$ is given by

$$
\left\langle f_{r * u} X, f_{r * u} Y\right\rangle=\left\langle F_{u}(r) X, F_{u}(r) Y\right\rangle
$$

and

$$
\begin{aligned}
\frac{d}{d t}\left\langle F_{u}(t) X, F_{u}(t) Y\right\rangle & =2\left\langle F_{u}^{\prime}(t) X, F_{u}(t) X\right\rangle \\
& =2\left\langle F_{u}^{\prime}(t) F_{u}(t)^{-1} F_{u}(t) X, F_{u}(t) X\right\rangle \\
& =-2\left\langle S_{u}(t) F_{u}(t) X, F_{u}(t) X\right\rangle .
\end{aligned}
$$

Also for small $t$ it is not hard to see

$$
F(t)=\left(\frac{1}{t}\right) I+O(1)
$$

Therefore the comparison result for tubes can be used to prove the inequality.

\section{Rigidity in Flat Spaces.}

Let $\mathbb{R}_{k}^{n}=\mathbb{R}_{k}^{n}(0)$ be the flat simply connected space form of index $k$. This is $\mathbb{R}^{n}$ with the semi-Riemannian metric

$$
\langle,\rangle=-\sum_{i=1}^{k} d x_{i}{ }^{2}+\sum_{i=k+1}^{n} d x_{i}{ }^{2} .
$$

Let $\eta$ be a unit vector in $\mathbb{R}_{k}^{n}$, that is $\langle\eta, \eta\rangle=+1$ or $\langle\eta, \eta\rangle=-1$. Let $\varepsilon=\langle\eta, \eta\rangle$, and let $\Lambda_{\eta}(\cdot)=\varepsilon\langle\cdot, \eta\rangle$. Then $\Lambda_{\eta}$ is the linear functional on $\mathbb{R}_{k}^{n}$. that has the orthogonal compliment of $\eta$ as kernel, and so that $\Lambda_{\eta}(\eta)=1$.

Definition 4.1. A subset $B \subset \mathbb{R}_{k}^{n}$ has the compact intersection property with respect to $\Lambda_{\eta}$ if and only if for each compact interval $[a, b] \subset \mathbb{R}$ the set $B \cap\left\{x: \Lambda_{\eta}(x) \in[a, b]\right\}$ has compact closure.

Theorem 4.2. Let $(\bar{M}, \bar{g})$ be a semi-Riemannian manifold of dimension $n \geq 3$ so that:

(1) The curvature tensor of $\bar{g}$ satisfies $\varepsilon \bar{R} \geq 0$ on $\bar{M}$ in the sense of Definition 1.1. 
(2) Every geodesic of sign $\varepsilon$ is complete. (That is if $\gamma:(a, b) \rightarrow \bar{M}$ is a geodesic with $\bar{g}\left(\gamma^{\prime}, \gamma^{\prime}\right)=\varepsilon$, then $\gamma$ extends to a geodesic defined on all of $\mathbb{R}$.)

Let $\eta_{0}$ be a unit vector in $\mathbb{R}_{k}^{n}$ of sign $\varepsilon$, and $B \subset \mathbb{R}_{k}^{n}$ be a closed subset so that $\mathbb{R}_{k}^{n} \backslash B$ is connected and

(3) $B \subset\left\{x: \Lambda_{\eta_{0}}(x)>0\right\}$

(4) $B$ has the compact intersection property with respect to $\Lambda_{\eta_{0}}$.

Then any local isometry $\varphi: \mathbb{R}_{k}^{n} \backslash B \rightarrow \bar{M}$ extents to a surjective local isometry $\widehat{\varphi}: \mathbb{R}_{k}^{n} \rightarrow \bar{M}$ defined on all of $\mathbb{R}_{k}^{n}$.

Remark 4.3. For Riemannian manifolds this does not lead to any results in the case of sectional curvatures $\leq 0$. However we note that a proof by Schroeder and Ziller [35, Theorem 1] of a result of Gromov [1, §5] leads to

Theorem. Let $(\bar{M}, \bar{g})$ be a complete simply connected Riemannian manifold of dimension at least $n \geq 2$ and non-positive sectional curvature. Let $m \geq n$ and $B \subset \mathbb{R}^{m}$ have the compact intersection property with respect to $\Lambda_{\eta}$ and assume $\mathbb{R}^{m} \backslash B$ is connected. Then any isometric imbedding $\varphi: \mathbb{R}^{n} \backslash B \rightarrow \bar{M}$ as a totally geodesic submanifold extends uniquely to a isometric imbedding $\widehat{\varphi}: \mathbb{R}^{m} \rightarrow \bar{M}$ as a totally geodesic submanifold.

This is interesting in that it is not assumed $B \subset\left\{x: \Lambda_{\eta}(x)>0\right\}$ or that the model space $\mathbb{R}^{m}$ and the space $\bar{M}$ have the same dimension. On the other hand it is important in their proof (which is based on Toponogov's triangle comparison theorem) that the map $\varphi$ be injective.

When $(\bar{M}, \bar{g})$ is Riemannian, the sectional curvatures of $(\bar{M}, \bar{g})$ are $\geq 0$, the set $B \subset \mathbb{R}^{n}$ is assumed compact and the map $\varphi: \mathbb{R}^{n} \backslash B \rightarrow \bar{M}$ is injective, the result can be deduced from a result of Greene and $\mathrm{Wu}$ [16, Theorem 1] (see also [1, Remark on p. 75]). When $\varphi$ is injective it is easy to see that the growth rate of the volume of geodesic balls is the same as that of balls in Euclidean space and so the Bishop-Gromov volume comparison theorem can be used to prove the result under the weaker assumption that the Ricci tensor of $(\bar{M}, \bar{g})$ is non-negative, cf. [23]. If $(\bar{M}, \bar{g})$ is a spin manifold and $\bar{M} \backslash \varphi\left[\mathbb{R}^{n} \backslash C\right]$ is compact then by rigidity results related to the positive mass conjecture it is enough to assume that the scalar curvature of $(\bar{M}, \bar{g})$ is non-negative (cf. [2]). If the dimension of $\bar{M}$ is $\leq 6$ then the proofs of Schoen and Yau $[32,34]$ of the positive mass conjecture imply the result without the assumption that $(\bar{M}, \bar{g})$ is spin. For other related rigidity results in the Riemannian case cf. [24] and [15]. 
The next corollary is not much more than a special case of Theorem 4.2, but in doing the inductive step of the proof of the theorem and as a lemma for use in later sections, it is helpful to have it stated separately.

Corollary 4.4. Let $D \subset \mathbb{R}_{k}^{n}$ be a connected domain with compact closure and smooth boundary and $\bar{g}$ be a semi-Riemannian metric on $D$ so that $\bar{g}$ agrees with the standard metric $g_{0}$ in a neighborhood of $\partial D$. If the curvature tensor satisfies either $\bar{R} \geq 0$ on all of $D$, or $\bar{R} \leq 0$ on all of $D$. Then $\bar{R} \equiv 0$.

Proof. Define a new semi-Riemannian metric $\bar{g}_{1}$ on $\mathbb{R}^{n}$ by letting $\bar{g}_{1}=\bar{g}$ in $D$ and $\bar{g}_{1}=g_{0}$ in $\mathbb{R}^{n} \backslash D$. As $\bar{g}$ and $g_{0}$ agree in a neighborhood of $\partial D$ this metric is smooth. We first consider the case $\bar{g}$, and thus $\bar{g}_{1}$, is positive definite. The hypotheses imply the sectional curvature of $\bar{g}_{1}$ is either nonnegative or non-positive. In these cases theorems of Greene and $\mathrm{Wu}[16$, Theorem 1] (for the case $\bar{R} \geq 0$ ) and Kasue and Sugahara [24, Theorem 2] (for the case $\bar{R} \leq 0$ ) imply that $\bar{R} \equiv 0$. The case that $\bar{g}$ is negative definite reduces to the positive definite case by replacing $\bar{g}$ by $-\bar{g}$. In all other cases it will be possible to choose a unit vector $\eta$ so that $\langle\eta, \eta\rangle \bar{R} \geq 0$. Then since $D$ is compact it will have the compact intersection property with respect to $\Lambda_{\eta}$. So this result follows from Theorem 4.2.

Proof of Theorem 4.2. The proof is by induction on the dimension $n$ of $\bar{M}$. The base of the induction is Theorem 2.9. Now some notation is needed. Let $N\left(\eta_{0}\right)=\left\{x \in \mathbb{R}_{k}^{n}: \Lambda_{\eta_{0}}(x)=0\right\}$ be the hyperplane through the origin, orthogonal to $\eta_{0}$, and set $M\left(\eta_{0}\right):=\varphi\left[N\left(\eta_{0}\right)\right]$. Let $\bar{\eta}_{0}$ be the unit normal field to $M\left(\eta_{0}\right)$ so that $\bar{\eta}_{0}(\varphi(0))=\varphi_{* 0} \eta_{0}$. For $r \in \mathbb{R}$ define $f_{\eta_{0}, r}: N\left(\eta_{0}\right) \rightarrow \bar{M}$ by

$$
f_{\eta_{0}, r}(x)=\exp _{\varphi(x)}\left(r \bar{\eta}_{0}(\varphi(x))\right)
$$

where exp is the exponential map of the metric $\bar{g}$. Thus $M\left(\eta_{0}\right)[r]:=$ $f_{\eta_{0}, r}\left[M\left(\eta_{0}\right)\right]$ is the parallel hypersurface to $M\left(\eta_{0}\right)$ at a distance $r$.

Our next goal is to show the curvature tensor $\bar{R}$ of $(\bar{M}, \bar{g})$ is zero at any point of the form $f_{\eta_{0}, r}(x)$. If $x \in N\left(\eta_{0}\right)$ and the segment $t \mapsto x+t \eta_{0} \notin B$ $(0 \leq t \leq r)$ then, as $\varphi$ is a local isometry on $\mathbb{R}_{k}^{n} \backslash B$, we have $\bar{R}_{f_{\eta_{0}, r}(x)}=0$, and also the image of $f_{\eta_{0}, r}$ near $f_{\eta_{0}, r}(x)$ is given by $\varphi\left\{y \in \mathbb{R}_{k}^{n}: \Lambda_{\eta_{0}}(y)=r\right\}$. Therefore near $f_{\eta_{0}, r}(x)$ the image of $f_{\eta_{0}, r}$ is totally geodesic and $f_{\eta, r}$ : $N\left(\eta_{0}\right) \rightarrow M\left(\eta_{0}\right)[r]$ is a local isometry near $x$. Set

$$
r_{0}=\sup \left\{r: \bar{R}_{f_{\eta_{0}, s}(x)}=0 \text { for all } x \in N\left(\eta_{0}\right) \text { and } s \leq r\right\} .
$$


If $r_{0}=\infty$, then $\bar{R}_{f_{\eta_{0}, r}(x)}=0$ for all $x \in N\left(\eta_{0}\right)$ and $r \in \mathbb{R}$ as claimed. Thus assume, toward a contradiction, $r_{0}<\infty$. From the hypothesis of the theorem $r_{0}>0$. If $r \leq r_{0}$ the above discussion shows $f_{\eta_{0}, r}: N\left(\eta_{0}\right) \rightarrow$ $M\left(\eta_{0}\right)[r]$ is a local isometry and so it is an immersion. By continuity if $r>r_{0}$ is only slightly larger than $r_{0}$ then $f_{\eta_{0}, r}: N\left(r_{0}\right) \rightarrow \bar{M}$ will also be an immersion. For such an $r$ consider the hypersurface $M\left(\eta_{0}\right)[r]=f_{\eta_{0}, r}\left[N\left(\eta_{0}\right)\right]$. By the compact intersection property there is a connected domain with smooth boundary $D_{0} \subset N\left(\eta_{0}\right)$ with compact closure and so that if $x \in$ $N\left(\eta_{0}\right) \backslash D_{0}$ and $s \leq r$ then $x+s \eta_{0} \notin B$. Therefore the pulled back metric $f_{\eta_{0}, r}^{*} \bar{g}$ is the usual flat metric on $N\left(\eta_{0}\right) \backslash D_{0}$.

We now use the comparison theorem. Using the Riccati equation satisfied by the Weingarten map of a family of parallel hypersurfaces (Proposition 2.3) and one of the comparison results for Riccati equations (Theorem 3.8), if $S_{f_{\eta_{0}, r}(x)}$ is the Weingarten map of $M(\eta)[r]$ at $f_{\eta_{0}, r}(x)$ then $\bigwedge^{2}\left(S_{f_{\eta_{0}, r}(x)}\right) \geq 0$. Let $R^{\eta_{0}, r}$ be the curvature tensor of $M\left(\eta_{0}\right)[r]$. Then by the Gauss curvature equation (2.2) multiplied by $\varepsilon$ and the assumptions on $\bar{R}$, we have

$$
\begin{aligned}
& \varepsilon\left\langle R^{\eta_{0}, r}(X, Y) Y, X\right\rangle \\
& \quad=\varepsilon\langle\bar{R}(X, Y) Y, X\rangle+\left\langle\bigwedge^{2}\left(S_{f_{\eta_{0}, r}(x)}\right) X \wedge Y, X \wedge Y\right\rangle \geq 0
\end{aligned}
$$

for any $X, Y$ tangent to $M(\eta)[r]$. Therefore the metric $f_{\eta_{0}, r}{ }^{*} \bar{g}$ agrees with the usual metric on $N\left(\eta_{0}\right)$ outside of $D_{0}$ and the curvature satisfies $\varepsilon\left\langle R^{\eta_{0}, r}(X, Y) Y, X\right\rangle \geq 0$. Thus by the induction hypothesis this implies $R^{\eta_{0}, r} \equiv 0$. For this to hold we must have

$$
\left\langle\bigwedge^{2}\left(S_{f_{\eta_{0}, r}(x)}\right) X \wedge Y, X \wedge Y\right\rangle=0
$$

for all $X, Y$ tangent to $M(\eta)[r]$. Putting this in the last equation implies $\langle\bar{R}(X, Y) Y, X\rangle=0$ for all $X, Y$ tangent to $M\left(\eta_{0}\right)[r]$. Unfortunately this is not enough to conclude directly that full curvature tensor $\bar{R}$ vanishes along $M\left(\eta_{0}\right)[r]$.

Let $y_{1}=f_{\eta_{0}, r_{1}}\left(x_{1}\right)$ be so that $\bar{R}_{y_{1}} \neq 0$. Assume $r_{1}$ is taken to only be slightly larger than $r_{0}$ so that $f_{\eta_{0}, r_{1}}$ is an immersion. Let $\eta \in \mathbb{R}_{k}^{n}$ be a unit vector. Then define $N(\eta)=\left\{x \in \mathbb{R}_{k}^{n}:\langle x, \eta\rangle=0\right\}$, etc. just as was done in the case of $N\left(\eta_{0}\right)$. Note however that in general $N(\eta) \cap B \neq \emptyset$ and so $M(\eta)=\varphi[N(\eta) \backslash B]$ and the maps $f_{\eta, r}$ are only defined on $N(\eta) \backslash B$. Let $D_{1} \subset N\left(\eta_{0}\right)$ be a connected domain with smooth boundary so that for all $r \leq r_{1}, B \cap\left(N\left(\eta_{0}\right)+r \eta_{0}\right)$ is a subset of the interiors of $D_{1}+r \eta_{1}$. Then the pulled back metric $f_{\eta_{0}, r_{1}}^{*} \bar{g}$ agrees with the standard flat metric of $N\left(\eta_{0}\right)$ on $\partial D_{1}$. Now let $\eta$ be a unit vector of $\mathbb{R}_{k}^{n}$ which is very close to $\eta_{0}$. Then 
for some $r$ very close to $r_{1}$ there holds $y_{1}=f_{\eta, r}(x)$ for some $x \in N(\eta)$. Note here that $r, x$ are functions of $\eta$.

If $\eta$ is close enough to $\eta_{0}$ there is a domain $D \subset N(\eta)$, which can be taken to be close to the domain $D_{1} \subset N\left(\eta_{0}\right)$, so that the pulled back metric $f_{\eta, r}^{*} \bar{g}$ agrees with the standard flat metric of $N(\eta)$ on a neighborhood of $\partial D$. The comparison argument of the last paragraph still holds and so the curvature tensor $R^{\eta, r}$ of $f_{\eta, r}^{*} \bar{g}$ satisfies $\varepsilon\left\langle R^{\eta, r}(X, Y) Y, X\right\rangle \geq 0$. Thus by the induction hypothesis and Corollary $4.4, R^{\eta, r} \equiv 0$ on $D$. Again this implies $\langle\bar{R}(X, Y) Y, X\rangle=0$ for all vectors $X, Y$ tangent to $f_{\eta, r}[D]$.

Let $\xi(\eta)$ be the normal to $f_{\eta, r(\eta)}[D]$ at $y_{1}$. Then the map $\eta \mapsto \xi(\eta) \in$ $T(\bar{M})_{y_{1}}$ is smooth in a neighborhood of $\eta_{0}$, and if we choose $r_{1}$ close enough to $r_{0}$ we also have that the derivative $\xi_{*}$ is non-singular at $\eta_{0}$. By the implicit function theorem this implies there is a neighborhood $U$ of $\xi\left(\eta_{0}\right)$ in the tangent space $T(\bar{M})_{y_{1}}$ so that if $u$ is a unit vector in $U$, then there is a unit vector $\eta$ near $\eta_{0}$ with $\xi(\eta)=u$. This in turn implies that if $X_{0}, Y_{0}$ are linearly independent vectors in

$$
T\left(f_{\eta_{0}, r_{1}}\left[N\left(\eta_{0}\right)\right]\right)_{y_{1}}=\xi\left(\eta_{0}\right)^{\perp}
$$

then there are neighborhoods $V$ of $X_{0}$ and $W$ of $Y_{0}$ in $T(\bar{M})_{f_{y_{1}}}$ so that if $X \in$ $V$ and $Y \in W$ then there is a $\eta$ near $\eta_{0}$ so that both $X$ and $Y$ are orthogonal to $\xi(\eta)$. Thus $X, Y \in \xi(\eta)^{\perp}=T\left(f_{\eta, r}[D]\right)$. Therefore by the discussion of the last paragraph $\langle\bar{R}(X, Y) Y, X\rangle=0$. The map $(X, Y) \mapsto\langle\bar{R}(X, Y) Y, X\rangle$ is a polynomial map on $T(\bar{M})_{y_{1}} \times T(\bar{M})_{y_{1}}$, and this map vanishes on the open set $V \times W$. Thus $\bar{R}_{y_{1}}=0$. However the point $y_{1}$ was chosen so that $\bar{R}_{y_{1}} \neq 0$. This contradiction completes the proof of the earlier claim that $\bar{R}=0$ at any point of the form $y=f_{\eta_{0}, r}$.

Note that points of $\mathbb{R}_{k}^{n}$ have a unique expression of the form $x+r \eta_{0}$ where $x \in N\left(\eta_{0}\right)=\eta_{0}^{\perp}$ and $r \in \mathbb{R}$. Define $\widehat{\varphi}: \mathbb{R}_{k}^{n} \rightarrow \bar{M}$ by

$$
\widehat{\varphi}\left(x+r \eta_{0}\right)=\exp _{\varphi(x)}\left(r \bar{\eta}_{0}(\varphi(x))\right)=f_{\eta_{0}, r}(x) .
$$

Using that $\bar{R}=0$ at all points of the form $f_{\eta_{0}, r}(x)$ it is not hard to show $\widehat{\varphi}$ is a local isometry. This gives the required extension of $\varphi$ to $\mathbb{R}_{k}^{n}$. That $\hat{\varphi}$ is surjective follows from Proposition 2.5.

\section{Rigidity in Simply Connected Space Forms.}

The results in this section are for Riemannian and Lorentzian manifolds. The central ideas are an inductive argument using parallel hypersurfaces and 
rigidity results for warped products that follow directly from the comparison theory.

\subsection{Rigidity Lemmas in Warped Products.}

Let $(M, g)$ be a Riemannian manifold and let $w:[0, L] \rightarrow(0, \infty)$ be a positive $C^{2}$ function with $w(0)=1$. Set $\bar{r}=-w^{\prime \prime} / w$ and $a=w^{\prime}(0)$. Then

$$
w^{\prime \prime}(t)+\bar{r}(t) w(t)=0, \quad w(0)=1, \quad w^{\prime}(0)=a .
$$

Let $\varepsilon=+1$ or $\varepsilon=-1$ and let $\bar{g}_{w}$ be the warped product metric

$$
\bar{g}_{w}:=w(t)^{2} g+\varepsilon d t^{2}
$$

on $\bar{M}:=M \times[0, L]$. Then for each $x \in M$ the curve $c_{x}(t):=(x, t)$ is a unit speed geodesic in $\left(M \times[0, L], \bar{g}_{w}\right)$. Then $\partial_{t}$ is a normal to the hypersurface $M \times\{t\}$ and with respect to this normal the Weingarten map is $S(t)=$ $-w^{\prime}(t) / w(t) I$. In particular the Weingarten map of $M \times\{0\}$ is $-w^{\prime}(0) I=a I$.

Definition 5.1. A semi-Riemannian metric $\bar{g}$ on $M \times[0, L]$ is adapted to the product structure if for each $x \in M$ the curve $c_{x}(t)$ is a unit speed geodesic with respect to the metric $\bar{g}$.

If $x^{1}, \ldots, x^{n-1}$ are local coordinates on $M$, then $x^{1}, \ldots, x^{n-1}, t$ are local coordinates on $M \times[0, L]$ and the metric $\bar{g}$ on $M \times[0, L]$ is adapted to the product structure if and only if the metric is of the form

$$
\bar{g}=\sum_{i, j=1}^{n-1} g_{i j}\left(x^{1}, \ldots, x^{n-1}, t\right) d x^{i} d x^{j}+\varepsilon d t^{2} .
$$

Remark 5.2. Let $f:(M, g) \rightarrow(\bar{M}, \bar{g})$ be an isometric immersion of $(M, g)$ into $(\bar{M}, \bar{g})$ as a hypersurface there is a globally defined unit normal $\eta$ along $f$. Define a map $F: M \times[0, L] \rightarrow \bar{M}$ by $F(x, t)=\exp (t \eta(x))$. If this is a local diffeomorphism then the Gauss lemma implies the metric $F^{*} \bar{g}$ is adapted to the product structure of $M \times[0, L]$.

If $\bar{g}$ is adapted to the product structure of $M \times[0, L]$ then $\partial_{t}$ is a unit normal to the hypersurfaces $M \times\{t\}$. Denote by $\bar{S}(t):=\bar{\nabla}_{X} \partial_{t}$ the Weingarten map of $M \times\{t\}$ in the metric $\bar{g}$. Along each of the geodesics $c_{x}(t)=(x, t)$ there and let $\bar{R}(t)=\bar{R}\left(\cdot, \partial_{t}\right) \partial_{t}$. 
Theorem 5.3. With the notation above (so that the restriction of the metric $\bar{g}$ to $M \times\{0\}$ is positive definite), assume that one of the two conditions

(1) $S(0) \geq a I$ on $M \times\{0\}$ and $\bar{R}(t) \geq \bar{r}(t) I$ on $M \times[0, L]$ or,

(2) $S(0) \leq a I$ on $M \times\{0\}$ and $\bar{R}(t) \leq \bar{r}(t) I$ on $M \times[0, L]$.

holds. If

$$
\bar{S}(L)=-\frac{w^{\prime}(L)}{w(L)} I \quad \text { on all of } \quad M \times\{L\}
$$

then $\bar{g} \equiv \bar{g}_{w}$.

Proof. Assume the first condition holds. The equality (5.1) implies that equality holds between $S_{1}(t):=-\frac{w^{\prime}(t)}{w(t)} I$ and $S_{2}(t):=\bar{S}(t)$ at $t=L$ in the comparison Theorem 3.2. This implies that $S_{1} \equiv S_{2}$ and $\bar{r}(t) I \equiv \bar{R}(t)$. The rest follows by a direct calculation.

Remark 5.4. For Theorem 3.2 to apply we need that the identity map on tangent spaces to $(M, g)$ is positive definite. This will only be the case when $g$ is positive definite, so that $(\bar{M}, \bar{g})$ will be Riemannian or Lorentzian. This is the reason why the results of this section are restricted to these cases.

Theorem 5.5. With the notation above assume that one of the conditions

(1) $S(0)=a I$ on $M \times\{0\}$ and $\bar{R}(t) \geq \bar{r}(t) I$ on $M \times[0, L]$, and $-\frac{w^{\prime}\left(r_{0}\right)}{w\left(r_{0}\right)}>0$,

(2) $S(0)=a I$ on $M \times\{0\}$ and $\bar{R}(t) \leq \bar{r}(t) I$ on $M \times[0, L]$, and $-\frac{w^{\prime}\left(r_{0}\right)}{w(L)}<0$,

(3) $S(0)=a I$ on $M=M \times\{0\}, \bar{R}(t) \leq \bar{r}(t) I$ and $\bar{S}(t)>0$ on $M \times[0, L]$, or

(4) $S(0)=a I$ on $M \times\{0\}, \bar{R}(t) \geq \bar{r}(t) I$ and $\bar{S}(t)<0$ on $M \times[0, L]$

holds. Then

$$
\bigwedge^{2}(S(L))=\left(\frac{w^{\prime}(L)}{w(L)}\right)^{2} \bigwedge^{2}(I) \quad \text { on all of } \quad M \times\{L\}
$$

implies $\bar{g} \equiv \bar{g}_{w}$. 
Proof. This follows from the comparison Theorems 3.9 and 3.10 in the same way that the last theorem follows from Theorem 3.2.

Remark 5.6. If the curvature tensor of $(\bar{M}, \bar{g})$ satisfies

$$
\bar{g}(\bar{R}(X, Y) Y, X) \geq \pm \varepsilon \bar{r}(t)\left(\bar{g}(X, X) \bar{g}(Y, Y)-\bar{g}(X, Y)^{2}\right)
$$

on all of the image of $G: M \times\left[0, r_{0}\right] \rightarrow \bar{M}$ then $\bar{R}(t) \geq \pm \bar{r}(t) I$. Likewise

$$
\bar{g}(\bar{R}(X, Y) Y, X) \leq \pm \varepsilon \bar{r}(t)\left(\bar{g}(X, X) \bar{g}(Y, Y)-\bar{g}(X, Y)^{2}\right)
$$

on the image of $G: M \times\left[0, r_{0}\right] \rightarrow \bar{M}$ implies $\bar{R}(t) \leq \pm \bar{r}(t) I$.

\subsection{The Rigidity Results.}

Definition 5.7. Let $N$ be a manifold and $\tau: N \rightarrow \mathbb{R}$ be a function. Then $B \subset N$ has the compact intersection property with respect to $\tau$ if for every compact interval $[a, b] \subset \mathbb{R}$ the set $\{x \in B: \tau(x) \in[a, b]\}$ has compact closure in $N$.

The rigidity results of this section are of the following type: Let $k=0$ or $k=1$ and let $\left(\mathbb{R}_{k}^{n}\left(K_{0}\right), g_{\mathbb{R}_{k}^{n}\left(K_{0}\right)}\right)$ be a model space (by which we mean one of the spaces listed in Table 2$)$ and $(\bar{M}, \bar{g})$ a semi-Riemannian manifold of the same dimension and index as $\mathbb{R}_{k}^{n}\left(K_{0}\right)$. Let $B \subset \mathbb{R}_{k}^{n}\left(K_{0}\right)$ be a closed set and $f:\left(\mathbb{R}_{k}^{n}\left(K_{0}\right) \backslash B\right) \rightarrow \bar{M}$ a local isometry. Then we are looking for conditions on $B$ and the curvature tensor of $(\bar{M}, \bar{g})$ so that $f$ will extend to a local isometry defined on all of $\mathbb{R}_{k}^{n}\left(K_{0}\right)$. The basic conditions are that $B$ not be too large (which will usually mean that it have the compact intersection property with respect to some function $\tau$ ), that $\mathbb{R}_{k}^{n}\left(K_{0}\right) \backslash B$ be connected (which is easily seen to be necessary) and that $(\bar{M}, \bar{g})$ is geodesically complete with respect to geodesics of some sign. Table 2 summarizes the conditions on the model space, the curvature bounds, the sign of the complete geodesics (column headed by Geo.), the exceptional set, and the function.

In the first two rows of the table $S^{n-1}$ is imbedded in $S^{n}=\mathbb{R}_{0}^{n}(+1)$ as an equator (that is as a totally geodesic submanifold). In the next two rows $\mathbb{R}_{0}^{n}(-1)$ is viewed as a warped product as in row 1 of Table 1 of Section 2.2 and the function $\tau$ is the warping function $w$. Likewise in the next two rows $B$ is a subset of the "half space" $\mathbf{H}_{1}^{n}(+1)$ in the de Sitter space $\mathbb{R}_{1}^{n}(+1)$ which is represented as a warped product in row 2 of Table 1 and in this case the function $\tau$ is again the warping function (see also Definition 2.6). The 
Table 2.

\begin{tabular}{|c|c|c|c|c|}
\hline Model & Curv. & Geo. & Exceptional Set & Function \\
\hline \hline$S^{n}=\mathbb{R}_{0}^{n}(+1)$ & $\bar{R} \geq+1$ & + & $B \subset S^{n} \backslash S^{n-1}$ & - \\
\hline $\mathbb{R}_{0}^{n}(-1)$ & $\bar{R} \geq-1$ & + & & - \\
\hline $\mathbb{R}_{1}^{n}(+1)$ & $\bar{R} \geq-1$ & + & $B \subset\{\tau>0\}$ & $\tau=w=e^{-t}$ \\
\hline $\mathbb{R}_{1}^{n}(+1)$ & $\bar{R} \leq+1$ & - & $B \subset\{\tau>0\} \subset \mathbf{H}_{1}^{n}(+1)$ & $\tau=w=e^{t}$ \\
\hline $\mathbb{R}_{1}^{n}(-1)$ & $\bar{R} \leq+1$ & - & $B \subset \mathbb{R}_{1}^{n}(+1) \backslash S^{n-1}$ & - \\
\hline $\mathbb{R}_{1}^{n}(-1)$ & $\bar{R} \geq-1$ & - & $B \subset\{\tau>0\}$ & $\begin{array}{l}\tau=\operatorname{time} \\
\text { function of } \\
\text { a geodesic }\end{array}$ \\
\hline
\end{tabular}

de Sitter space $\mathbb{R}_{1}^{n}(+1)$ has a another representation as a warped product $-d t^{2}+\cosh ^{2}(t) \bar{g}_{\mathbf{R}_{0}^{n-1}(+1)}$ and thus $S^{n-1}=\mathbb{R}_{0}^{n-1}(+1)$ is being viewed as the submanifold $S^{n-1} \times\{0\}$ of $\mathbb{R}_{1}^{n}(-1)$. In the first two rows for the anti-de Sitter space $\mathbb{R}_{1}^{n}(-1)$, the function $\tau$ is the time function of a timelike unit speed geodesic as in Definition 2.8. Finally in the last two rows of the table $B$ is a subset of "strip" $\mathbf{S}_{1}^{n}(-1)$ in the anti-de Sitter space as in Definition 2.7 and the function $\tau$ is the warping function.

Theorem 5.8. Let $\left(\mathbb{R}_{k}^{n}\left(K_{0}\right), g_{\mathbb{R}_{k}^{n}\left(K_{0}\right)}\right)$ be one of the model spaces in Table 2 of dimension at least three and let $B \subset \mathbb{R}_{k}^{n}\left(K_{0}\right)$ be a closed set so that $\mathbb{R}_{k}^{n}\left(K_{0}\right) \backslash B$ is connected. Then for any of the twelve rows in the table if $B$ is a subset of the indicated set and if a function $\tau$ is given in the last column assume that $B$ has the compact intersection property with respect to $\tau$. Let $(\bar{M}, \bar{g})$ be a semi-Riemannian manifold of the same dimension and index as $\left(\mathbb{R}_{k}^{n}\left(K_{0}\right), g_{\mathbb{R}_{k}^{n}\left(K_{0}\right)}\right)$ so that the curvature tensor $\bar{R}$ of $(\bar{M}, \bar{g})$ satisfies the indicated inequality and that every geodesic of $(\bar{M}, \bar{g})$ of the indicated sign is complete. Then any local isometry $\varphi: \mathbb{R}_{k}^{n}\left(K_{0}\right) \backslash B \rightarrow \bar{M}$ extends to 
a surjective local isometry $\widehat{\varphi}: \mathbb{R}_{k}^{n}\left(K_{0}\right) \rightarrow \bar{M}$. Thus $(\bar{M}, \bar{g})$ has constant curvature.

Remark 5.9. (1) In the case of $\mathbb{R}_{0}^{n}(-1)$ (the Riemannian hyperbolic space), when $B$ is compact, $\varphi: \mathbb{R}_{1}^{n}(-1) \backslash B \rightarrow \bar{M}$ is injective, and $\bar{M}$ is simply connected (and when $\bar{R} \geq 0$ also assume that $(\bar{M}, \bar{g})$ has a pole, that is a point where $\exp _{x_{0}}: T(\bar{M})_{x_{0}} \rightarrow \bar{M}$ is a diffeomorphism) the result can be deduced from a result of Kasue and Sugahara [24, page 697]. If $\bar{R} \leq-1, \bar{M}$ is simply connected and

$$
\bar{M} \backslash \varphi\left[\mathbb{R}_{0}^{n}(-1) \backslash B\right]
$$

is compact this is also a special case of a result of Schroeder and Ziller [35, Theorem 7]. We note that our result allows the set $B$ to be non-compact and does not require $\varphi$ to be injective.

For the space $\mathbb{R}_{0}^{n}(+1)=S^{n}$ when $\bar{R} \geq+1$ if the space $\bar{M} \backslash \varphi\left[S^{n} \backslash B\right]$ is simply connected, compact and strictly convex then the result is covered by the result of Schroeder and Ziller [35, Theorem 7]. In the case of $\mathbb{R}_{0}^{n}(+1)=S^{n}$ and $\bar{R} \leq+1$ to the best of our knowledge the result is new.

(2) Because of the failure of Theorems 5.3 and 5.5 when $(\bar{M}, \bar{g})$ is not Riemannian or Lorentzian, the method of proof here fails as a method for proving rigidity theorems for semi-Riemannian manifolds of arbitrary index.

The proofs all follow the same pattern: An induction on dimension using hypersurfaces parallel to a standard hypersurface and the warped product rigidity results of Section 5.1. We will do one case in detail and leave the others to the reader. To avoid inessential but annoying problems involving signs, the case we consider is the Riemannian case where all the geometric complications involved occur.

Theorem 5.10. Let $(\bar{M}, \bar{g})$ be a complete Riemannian manifold of dimension $n \geq 3$ with sectional curvatures $\leq 1$. Let $B \subset S^{n} \backslash S^{n-1}$ be a closed set with $S^{n} \backslash B$ connected and let $\varphi: S^{n} \backslash B \rightarrow \bar{M}$ be a local isometry. Then $\varphi$ extends to a surjective local isometry $\widehat{\varphi}: S^{n} \rightarrow \bar{M}$.

In doing the induction step it is useful to have the following special case. The notation is as follows: $\left(S^{n-1} \times\left(-r_{0}, r_{0}\right), g_{\mathbf{S}}\right)$ is the tube of radius $r_{0}$ about the equator $S^{n-1}$. 
Corollary 5.11. Let $(\bar{M}, \bar{g})$ be a connected complete Riemannian manifold of dimension at least three and so that the sectional curvature of $(\bar{M}, \bar{g})$ satisfies $K_{\bar{M}} \leq 1$. Let $0<r_{0}<\pi / 2$. If $(\bar{M}, \bar{g})$ contains a subset isometric to $\left(S^{n-1} \times\left(-r_{0}, r_{0}\right), g_{\mathbf{S}}\right)$, then $(\bar{M}, \bar{g})$ is isometric to $\left(S^{n}, g_{\mathbf{S}}\right)$.

Proof. By assumption there is an isometry $\varphi:\left(S^{n-1} \times\left(-r_{0}, r_{0}\right), g_{\mathbf{S}}\right) \rightarrow$ $(\bar{M}, \bar{g})$. By the theorem this extends to a local isometry $\widehat{\varphi}:\left(S^{n}, g_{\mathbf{S}}\right) \rightarrow$ $(\bar{M}, \bar{g})$. As $\widehat{\varphi}$ is a local isometry the image $\widehat{\varphi}\left[S^{n}\right]$ is open in $\bar{M}$. But as $S^{n}$ is compact the image is also closed. As $\bar{M}$ is connected this implies $\widehat{\varphi}$ is surjective. To show $\widehat{\varphi}$ is an isometry it only remains to show it is injective.

Note $\widehat{\varphi}: S^{n} \rightarrow \bar{M}$ is a covering map. Let $G$ be the group of deck transformations of $\widehat{\varphi}: S^{n} \rightarrow \bar{M}$, that is $G$ is the set of maps $a: S^{n} \rightarrow S^{n}$ so that $\widehat{\varphi} \circ a=\widehat{\varphi}$. If $a \in G$, then $a: S^{n} \rightarrow S^{n}$ is an isometry of $g_{\mathbf{S}}$. It follows $a\left[S^{n-1}\right] \cap S^{n-1} \neq \emptyset$. Let $x \in S^{n-1}$ so that $a(x) \in S^{n-1}$. Then $\widehat{\varphi}(a x)=\widehat{\varphi}(x)$. But on $S^{n-1}$ we have $\widehat{\varphi}=\varphi$ so $\varphi(a x)=\varphi(x)$. But $\varphi$ is an isometry and therefore injective. Thus $a(x)=x$. But a deck transformation with a fixed point is the identity so $a=$ Identity. As $a$ was an arbitrary element of $G$ this implies $G$ is trivial. As $S^{n}$ is simply connected this implies $\widehat{\varphi}$ is injective and completes the proof $(\bar{M}, \bar{g})$ is isometric to $\left(S^{n}, g_{\mathbf{S}}\right)$.

Proof of Theorem 5.10. It is enough to show $(\bar{M}, \bar{g})$ has constant sectional curvature 1 . For then the universal covering space of $(\bar{M}, \bar{g})$ is the standard sphere $\left(S^{n}, g_{\mathbf{S}}\right)$. Let $\pi: S^{n} \rightarrow \bar{M}$ be the covering map. As any local isometry between connected open sets in $S^{n}$ extends to a global isometry there is an isometry $\psi: S^{n} \rightarrow S^{n}$ so that $\left.\pi \circ \psi\right|_{S^{n} \backslash B}=\varphi$. Then $\widehat{\varphi}:=\pi \circ \psi$ is the required extension. That $\widehat{\varphi}$ is surjective follows from Proposition 2.5 .

The rest of the proof is devoted to showing that $(\bar{M}, \bar{g})$ has constant sectional curvature 1 . Let $B$ be the set of points $x$ of $\bar{M}$ where some sectional curvature at $x$ is less than +1 . Toward a contradiction assume $B \neq \emptyset$. We may assume $r_{0}$ is maximal with respect to the property that there is a local isometry $\varphi:\left(S^{n-1} \times\left[-r_{0}, r_{0}\right], g_{\mathrm{S}}\right) \rightarrow(\bar{M}, \bar{g})$. For $U \subset \bar{M}$ let $\mathrm{Cl}(U)$ be the closure of $U$ in $\bar{M}$. Then as $r_{0}$ is maximal there is a point $y_{0} \in$ $\varphi\left[S^{n-1} \times\left[-r_{0}, r_{0}\right]\right] \cap \mathrm{Cl}(B)$. By acting with an isometry on $S^{n-1} \times\{0\}$ we may choose the foliation defined by $S^{n-1} \times\{r\}$ so that in fact $y_{0}=$ $\varphi\left[S^{n-1} \times\left[-r_{0}, r_{0}\right]\right] \cap \mathrm{Cl}(B)$. Let $x_{0} \in S^{n-1} \times\left[-r_{0}, r_{0}\right]$ so that $\varphi\left(x_{0}\right)=y_{0}$. We may assume $x_{0} \in S^{n-1} \times\left\{r_{0}\right\}$. If $x_{0} \in S^{n-1} \times\left\{-r_{0}\right\}$ then do the change of variable $(x, t) \mapsto(x,-t)$ on $S^{n-1} \times\left[-r_{0}, r_{0}\right]$. 
Let $K_{0}=1 / \cos ^{2}\left(r_{0}\right)$ so that $K_{0}$ is the sectional curvature of $S^{n-1} \times\left\{r_{0}\right\}$ with the metric induced by $g_{\mathbf{S}}$. Let $0<r_{1}<r_{0}$ and let $K_{1}=1 / \cos ^{2}\left(r_{1}\right)$.

Let $S^{n-1}\left(K_{1}\right)$ be the totally umbilic submanifold of $\left(S^{n-1} \times\left[-r_{0}, r_{1}\right]\right)$ which is tangent to $S^{n-1} \times\left\{r_{0}\right\}$ at the point $x_{0}$. Then $S^{n-1}\left(K_{1}\right) \cap S^{n-1} \times$ $\left\{r_{0}\right\}=\left\{x_{0}\right\}$ and the Weingarten map of $S^{n-1}\left(K_{1}\right)$ is $S \equiv\left(\sin \left(r_{1}\right) / \cos \left(r_{1}\right)\right) I$ (cf. Table 1). If $r_{1}$ is chosen close enough to $r_{0}$ then $S^{n-1}\left(K_{1}\right) \subset S^{n-1} \times$ $\left[0, r_{0}\right]$.

Let $\eta$ be the unit normal along $S^{n-1}\left(K_{1}\right)$ so that $\eta\left(x_{0}\right)=\partial_{t x_{0}}$. Let $r_{3}$ be a small positive number to be chosen later and define a map $G$ : $S^{n-1}\left(K_{1}\right) \times\left[0, r_{3}\right] \rightarrow \bar{M}$ by

$$
G(x, t)=\exp _{\varphi(x)}\left(\varphi_{*} \eta(x)\right) .
$$

As $\varphi$ is a local isometry on $S^{n-1} \times\left[-r_{0}, r_{0}\right]$ the submanifold $\varphi\left[S^{n-1}\left(K_{1}\right)\right]$ is also totally umbilic in $\bar{M}$ with Weingarten $\operatorname{map}\left(\sin \left(r_{1}\right) / \cos \left(r_{1}\right)\right) I$. For $t>0$ let $S_{t}$ be the Weingarten map of $S^{n-1}\left(K_{1}\right) \times\{t\}$ in $S^{n-1}\left(K_{1}\right) \times\left[0, r_{3}\right]$ with respect to the pullback metric $G^{*} \bar{M}$. If $u(t):=\tan \left(r_{1}+t\right)$ then $u^{\prime}=u^{2}+1$. By the assumption on the curvature and the comparison Theorem 3.10,

$$
\bigwedge^{2}\left(S_{t}\right) \leq u(t)^{2} \bigwedge^{2}(I) \text { on }\left[0, r_{3}\right]
$$

This, the assumption that the sectional curvature of $\bar{g}$ (and thus also the pulled back metric $G^{*} \bar{g}$ ) has sectional curvature $\leq 1$ implies the sectional curvature of the hypersurface $S^{n-1}\left(K_{1}\right) \times\left\{r_{3}\right\}$ with the metric induced by $G^{*} \bar{g}$ satisfies

$$
K_{S^{n-1}\left(K_{1}\right) \times\left\{r_{3}\right\}} \leq \frac{1}{\cos ^{2}\left(r_{1}+r_{3}\right)} .
$$

Let $K_{3}:=1 / \cos ^{2}\left(r_{1}+r_{3}\right)$. We now claim if $r_{3}>0$ is small enough, then equality holds in inequality (5.2). This follows from the theorem we are proving by induction. The base case is $n=3$ so that $S^{n-1}\left(K_{1}\right) \times\left\{r_{3}\right\}$ is two dimensional. Let

$$
\rho\left(r_{3}\right):=1 / \cos \left(r_{1}+r_{3}\right) .
$$

By making $r_{3}$ small enough, the Gauss curvature $K$ of the rescaled metric $\left(S^{2}\left(K_{1}\right) \times\left\{r_{3}\right\}, \rho\left(r_{3}\right) G^{*} \bar{g}\right)$ satisfies $K \leq 1$ and can be made arbitrarily close to 1 .

Also by making $r_{3}$ small we can make the set where $K=1$ as large as we please, in the sense that there is a subset of $\left(S^{2}\left(K_{1}\right) \times\left\{r_{3}\right\}, \rho\left(r_{3}\right)^{2} G^{*} \bar{g}\right)$ which is isometric to a standard sphere of constant Gauss curvature +1 with 
a very small ball deleted. Thus the two dimensional rigidity result Theorem 2.12 implies $\left(S^{2}\left(K_{1}\right) \times\left\{r_{3}\right\}, \rho\left(r_{3}\right) G^{*} \bar{g}\right)$ is isometric to a unit sphere. Thus the curvature of $\left(S^{2}\left(K_{1}\right) \times\left\{r_{3}\right\}, G^{*} \bar{g}\right)$ is identically $K_{3}$. The case of $n \geq 4$ is easier. Once $r_{3}$ is small enough that $\left(S^{n-1}\left(K_{1}\right) \times\left\{r_{3}\right\}, G^{*} \bar{g}\right)$ contains an open set which in turn contains a closed set isometric to a hemisphere in the standard sphere of constant sectional curvature $K_{3}$, induction and Corollary 5.11 implies $\left(S^{n-1}\left(K_{1}\right) \times\left\{r_{3}\right\}, G^{*} \bar{g}\right)$ has constant sectional curvature $K_{3}$. Thus equality holds in (5.2) as claimed.

But if equality holds in (5.2), then it follows, using that the sectional curvature of $(\bar{M}, \bar{g})$ is $\leq 1$ and the Gauss curvature equation, that $\bigwedge^{2}\left(S_{r_{3}}\right) \equiv$ $u\left(R_{3}\right)^{2} \bigwedge^{2}(I)$. Then Theorem 5.5 implies

$$
G:\left(\bigcup\left\{S^{n-1}\left(K_{1}\right) \times\{s\}: 0 \leq s \leq r_{3}\right\}, g_{\mathbf{S}}\right) \rightarrow(\bar{M}, \bar{g})
$$

is a local isometry. As the point $y_{0}=\varphi\left(x_{0}\right)$ is in the interior of the set

$$
G\left[\bigcup\left\{S^{n-1}\left(K_{1}\right) \times\{s\}: 0 \leq s \leq r_{3}\right\}\right] \cup \varphi\left[S^{n-1} \times\left[-r_{0}, r_{0}\right]\right]
$$

and both $\varphi$ and $G$ are local isometries this implies the sectional curvature of $(\bar{M}, \bar{g})$ is identically +1 in a neighborhood of $y_{0}$. But this contradicts the choice of $y_{0} \in \mathrm{Cl}(B)$ and completes the proof the sectional curvature of $(\bar{M}, \bar{g})$ is identically +1 . This in turn completes the proof of the theorem.

\subsection{Examples.}

We give some examples to show that at least with regard to the size of the sets $B$ in the results of Sections 5.2 and 4 the results are close to optimal. However in light of the many results $[4,8,9,14,17,27,29]$ to the effect that a manifold with indefinite metric, satisfying a one sided bound on the sectional curvature $\left(=\langle R(X, Y) Y, X\rangle /\left(\langle X, X\rangle\langle Y, Y\rangle-\langle X, Y\rangle^{2}\right)\right.$, must have constant sectional curvature, it is worth first giving examples to show that there are large numbers of semi-Riemannian manifolds that have one sided curvature bounds in our sense.

Let $\left(M_{1}, g_{1}\right)$ and $\left(M_{2}, g_{2}\right)$ be complete Riemannian manifolds and set $M=M_{1} \times M_{2}, g=g_{1}-g_{2}$. Then $(M, g)$ is a geodesically complete semiRiemannian manifold of index $k=\operatorname{dim} M_{2}$. If the sectional curvatures of $\left(M_{1}, g_{1}\right)$ are $\geq 0$ and the sectional curvatures of $\left(M_{2}, g_{2}\right)$ are $\leq 0$ then the curvature of $(M, g)$ satisfies $R \geq 0$. (The curvature of $-g$ will satisfy $R \leq 0$.)

For a related example let $\left(M_{1}, g_{1}\right)$ be a Riemannian manifold and let $\left(\mathbf{H}^{k}, g_{\mathbf{H}}\right)$ be the hyperbolic space of constant sectional curvature -1 . Let 
$\rho: \mathbf{H}^{k} \rightarrow[0, \infty)$ be the Riemannian distance from some point $x_{0} \in \mathbf{H}^{k}$. Let $M=M_{1} \times \mathbf{H}^{k}$ and let $g$ be the warped product metric $g=\cosh ^{2}(\rho) g_{1}-$ $g_{\mathbf{H}}$. If $\left(M_{1}, g_{1}\right)$ is compact with sectional curvatures $\geq+1$ then $(M, g)$ is geodesically complete and has curvature $R \geq 1$. (The metric $-g$ has curvature $\leq-1$.)

To get some more interesting examples we first consider the two dimensional case. Recall (cf. Section 2.3) that if $(M, g)$ is two dimensional the Gaussian curvature $K$ is defined by $K=g(R(X, Y) Y, X)$ where $X, Y$ is an orthonormal basis of $T(M)$. The proof of the following is straightforward.

Proposition 5.12. Let $\varepsilon_{1}, \varepsilon_{2}$ be \pm 1 and let $g_{0}=\varepsilon_{1} d x^{2}+\varepsilon_{2} d y^{2}$ be the standard flat metric on $\mathbb{R}^{2}$. Then there are geodesically complete metrics $g_{+}$ and $g_{-}$on $\mathbb{R}^{2}$, with Gaussian curvatures $K_{+}$and $K_{-}$respectively, so that $g_{ \pm}=g_{0}$ on the set $\{y \leq 1\}, K_{+} \geq 0, K_{-} \leq 0$ and neither of $K_{+}$or $K_{-}$is identically 0 .

By considering product metrics

$$
\left(\mathbb{R}_{j}^{n-2} \times \mathbb{R}^{2}, g_{\mathbb{R}_{j}^{n-2}}+g_{ \pm}\right)
$$

we see that for each $l$ there are geodesically complete metrics $g=g_{\mathbb{R}_{j}^{n-2}}+g_{ \pm}$ on $\mathbb{R}^{n}$ that agree with the standard metric

$$
g_{k}^{n}=-\sum_{i=1}^{k} d x_{i}^{2}+\sum_{i=k+1}^{n} d x_{i}^{2},
$$

on the set $\left\{x_{l} \leq 1\right\}$, whose curvature satisfies $R \geq 0$ (or $R \leq 0$ ) but which are not globally flat. In this case the exceptional set $B=\left\{x_{l} \geq 1\right\}$ does not satisfy the compact intersection property with respect to any $\Lambda_{\eta}$ which shows this condition is necessary in Theorem 4.2.

We note that in the Lorentzian case, the metrics constructed above do not satisfy the dominant energy condition. This is a consequence of the following result. Let $T_{\Lambda}=\mathrm{Ric}-\frac{1}{2} \mathrm{Scal} g+\Lambda g$. Then the Lorentzian manifold $(M, g)$ satisfies the dominant energy condition $[18, \S 4.3]$ if $T_{\Lambda}(X, X) \geq 0$ and $Y \mapsto T_{\Lambda}(X, Y)$ defines a nonspacelike covector for all nonspacelike $X, Y$.

Theorem 5.13. Let $(\bar{M}, \bar{g})$ be a timelike geodesically complete globally hyperbolic Lorentzian manifold of dimension n, satisfying the dominant energy condition with cosmological constant $\Lambda=\frac{-(n-1)(n-2)}{2} K_{0}$ for $K_{0} \geq 0$. Then, if there is an isometric immersion of $\mathbb{R}_{0}^{n-1}\left(K_{0}\right)$ as a totally geodesic hypersurface of $\bar{M}$, there is a surjective local isometry $\varphi:\left(\mathbb{R}_{1}^{n}\left(K_{0}\right)\right) \rightarrow \bar{M}$. 
Proof. In case $K_{0} \geq 0, \mathbb{R}_{1}^{n}\left(K_{0}\right)$ is globally hyperbolic and timelike geodesically complete. Hence the result follows from the conservation theorem (cf. [18, p. 94]) and uniqueness theorems for Einstein's equations (cf. [18, Ch. 7] or [38, Ch. 10]).

Remark 5.14. In case $K_{0}>0$, the condition that $(\bar{M}, \bar{g})$ is globally hyperbolic is not necessary in Theorem 5.13. The examples constructed above are globally hyperbolic. Therefore Theorem 5.13 implies there is a geodesically complete Lorentzian metric $g$ on $\mathbb{R}^{n}$ that agrees with the flat metric $-d x_{1}^{2}+d x_{2}^{2}+\cdots+d x_{n}^{2}$ on the set $\left\{x_{1} \leq 1\right\}$, whose curvature satisfies $R \geq 0$, but which is not flat. Then this metric will also satisfy the strong energy condition $\operatorname{Ric}(T, T) \geq 0$ for all timelike vectors $T$. However by Theorem 5.13 the dominant energy condition (with $\Lambda=0$ ) cannot hold on all of $\left(\mathbb{R}^{n}, g\right)$.

Several of the other model spaces we consider are warped products. To be concrete consider $\bar{g}=e^{2 t} g_{0}+d t^{2}$ where $g_{0}$ is the flat positive definite metric on $\mathbb{R}^{n-1}$. Then $\bar{g}$ is the metric on the hyperbolic space with constant sectional curvature -1 . Choose a smooth function $w(t)$ on $\mathbb{R}$ so that $w(t)=$ $e^{t}$ for $t \leq 1$ and $-\frac{w^{\prime \prime}(t)}{w(t)}<-1$, and $-\frac{w^{\prime}(t)}{w(t)}<-1$ for $t>1$ (such functions are not hard to construct). Then the curvature $\bar{R}_{1}$ of the metric $\bar{g}_{1}:=$ $w(t)^{2} g_{0}+d t^{2}$ will satisfy $\bar{R}_{1} \leq-1$ but $\bar{g}_{1}$ is not isometric to the metric $\bar{g}$ even though they agree on the set $\{t \leq 1\}$. In this case the exceptional set we are trying to extend across is $B=\{t \geq 1\}$ and this does not have the compact intersection property with respect to the warping function $t$. In this case it is also possible to find functions so that $w(t)=e^{t}$ for $t \leq 1$ and $-\frac{w^{\prime \prime}(t)}{w(t)}>-1$, and $-\frac{w^{\prime}(t)}{w(t)}>-1$ for $t>1$ which gives examples with $\bar{R}_{1} \geq-1$. Other examples relevant to Theorem 5.8 can be constructed along the same lines.

\section{Applications.}

\subsection{Ends of Constant Curvature and Rigidity.}

In this section we find all the geodesically complete ends of constant sectional curvature and with finite fundamental group. This can be combined with our earlier rigidity results to prove rigidity results for semi-Riemannian manifolds with an end of constant sectional curvature and finite fundamental group. 


\subsubsection{Structure of Ends of Constant Curvature.}

We first remark that in the case of flat ends of complete Riemannian manifolds there is a structure theory due to Eschenburg and Schroeder [10] that gives a complete classification of the locally Euclidean ends. It would be interesting to have a corresponding structure theory in the case of ends with constant sectional curvature -1 . There does not seem to be much known in this case except when the fundamental group of the end is finite and then Theorem 6.3 below applies.

Let $\left(\mathbb{R}_{k}^{n}\left(K_{0}\right), g_{\mathbb{R}_{k}^{n}\left(K_{0}\right)}\right)$ be one of our model spaces and let $G$ be a finite group of isometries acting on $\left(\mathbb{R}_{k}^{n}\left(K_{0}\right), g_{\mathbb{R}_{k}^{n}\left(K_{0}\right)}\right)$. Assume that there is a compact set $C \subset \mathbb{R}_{k}^{n}\left(K_{0}\right)$ so that $G$ is fixed point free on $\mathbb{R}_{k}^{n}\left(K_{0}\right) \backslash C$. (That is if $a \in G, a \neq 1$ then $a(x) \neq x$ for all $x \in \mathbb{R}_{k}^{n}\left(K_{0}\right) \backslash C$. In this case we say that $G$ is fixed point free on the complement of a compact set. As the group $G$ is finite by replacing $C$ with $\bigcup_{a \in G} a[C]$ we can assume that $C$ is setwise invariant under the action of $G$. Then the quotient space $G \backslash\left(\mathbb{R}_{k}^{n}\left(K_{0}\right) \backslash C\right)$ is a semi-Riemannian manifold with constant sectional curvature. We will show that all ends of constant curvature and finite fundamental group are isometric to examples of this type and give more information about what groups $G$ can be realized and how these groups act on $\left(\mathbb{R}_{k}^{n}\left(K_{0}\right), g_{\mathbb{R}_{k}^{n}\left(K_{0}\right)}\right)$.

Let $k$ be an integer, $0 \leq k \leq n$ and let $\mathbb{R}_{k}^{n}=\mathbb{R}_{k}^{n}(0)$ be the geodesically complete flat space form of index $k$. For $k>0$, write the tangent space to $\mathbb{R}_{k}^{n}$ as $T\left(\mathbb{R}_{k}^{n}\right)_{0}=\mathbb{R}_{k}^{k} \oplus \mathbb{R}_{0}^{n-k}$ and let $S^{k-1} \times S^{n-k-1}$ be the subset of $T\left(\mathbb{R}_{k}^{n}\right)_{0}=\mathbb{R}_{k}^{k} \oplus \mathbb{R}_{0}^{n-k}$ defined by $\{(x, y):\langle x, x\rangle=-1,\langle y, y\rangle=1\}$. We view $S^{k-1} \times S^{n-k-1}$ as the Riemannian product of spheres.

Let $O(k, n-k)$ denote the group of isometries of $\mathbb{R}_{k}^{n}$ fixing the origin. This can be identified with the group of linear isometries of the underlying indefinite inner product space. Note that any Riemannian isometry $a$ of $S^{k-1} \times S^{n-k-1}$ which preserves the product structure (i.e. $a \in O(k) \times O(n-$ $k)$ ) is induced by a unique $g \in O(k, n-k)$, and the action of the derivative $g_{*}$ on the tangent space $\mathbb{R}_{k}^{n} \oplus \mathbb{R}_{0}^{n-k}$ preserves the splitting and induces the mapping $a$ on $S^{k-1} \times S^{n-k-1}$. Thus any subgroup $G$ of $O(k) \times O(n-k)$ extends to to a subgroup of $O(k, n-k)$.

Call a subgroup $G$ of $O(k) \times O(n-k)$ strongly fixed point free if for every $a=\left(a_{1}, a_{2}\right) \in G$ if there is a point $u_{1} \in S^{k}$ with $a_{1} u_{1}=u_{1}$, or a point $u_{2} \in S^{n-k}$ with $a_{2} u_{2}=u_{2}$ then both $a_{1}$ and $a_{2}$ are the identity. That is $G$ is strongly fixed point free iff both the induced actions on $S^{k}$ and $S^{n-k}$ are fixed point free. 
It is easy to check that if $G$ is a subgroup of $O(k) \times O(n-k)$ then the induced action of $G$ on $\mathbb{R}_{k}^{n}$ is fixed point free on $\mathbb{R}_{k}^{n} \backslash\{0\}$ iff the action is strongly fixed point free. If $G$ is finite and strongly fixed point free, then we can form the orbit space $G \backslash \mathbb{R}_{k}^{n}$. This is a smooth manifold except at the orbit corresponding to the origin which is a conical singular point.

Definition 6.1. Let $G \subset O(k) \times O(n-k)$ be a finite group of isometries of the space $S^{k-1} \times S^{n-k-1}$ which is strongly fixed point free. Then the standard end of $\mathbb{R}_{k}^{n}$ determined by $G$ is the end of the space $G \backslash \mathbb{R}_{k}^{n}$ constructed above.

Let $K_{0}>0$. To make notation easier normalize so that $K_{0}=1$. Let $\left(\mathbf{H}^{k}, g_{\mathbf{H}}\right)$ be the hyperbolic space with constant sectional curvature -1 and let $\left(S^{n-k}, g_{\mathbf{S}}\right)$ be the standard sphere. Choose a point $x_{0} \in \mathbf{H}^{k}$ to use as an origin and let $\rho: \mathbf{H}^{k} \rightarrow[0, \infty)$ be the Riemannian distance from $x_{0}$.

For $1 \leq k<n-1$, the space $\mathbb{R}_{k}^{n}(+1)$ has a representation as the warped product

$$
\left(S^{n-k} \times \mathbf{H}^{k}, \cosh ^{2}(\rho) g_{\mathbf{S}}-g_{\mathbf{H}}\right) .
$$

Let $S^{k-1}$ be the unit sphere of $T\left(\mathbf{H}^{k}\right)_{x_{0}}$. Any isometry $a$ of $S^{k-1}$ is induced by a unique isometry of $\mathbf{H}^{k}$ fixing the origin $x_{0}$. Thus any isometry $a=$ $\left(a_{1}, a_{2}\right) \in O(n-k+1) \times O(k)$ of $S^{n-k} \times S^{k-1}$ induces an isometry of $\mathbb{R}_{k}^{n}(+1)=\left(S^{n-k} \times \mathbf{H}^{k}, \cosh ^{2}(\rho) g_{\mathbf{S}}-g_{\mathbf{H}}\right)$ in the obvious way.

If $G \subset O(n-k+1) \times O(k)$ we say $G$ is weakly fixed point free iff for any non-identity $\left(a_{1}, a_{2}\right) \in O(n-k+1) \times O(k)$ if $a_{2}$ fixes a point of $S^{k-1}$, then $a_{1}$ has no fixed points on $S^{n-k}$. Note that if $G \subset O(n-k+1) \times O(k)$ is a finite group, then the induced action on $\mathbb{R}_{k}^{n}(+1)$ is fixed point free on the complement of some compact set iff $G$ is weakly fixed point free. Thus the quotient space $G \backslash \mathbb{R}_{k}^{n}(+1)$ is smooth away form a compact set.

Finally consider the case of $k=n-1$, and let $x_{0}$ be a point of $\mathbb{R}_{n-1}^{n}\left(K_{0}\right)$ with $K_{0}>0$. Let $\mathbb{Z}_{2}$ act on $\mathbb{R}_{n-1}^{n}\left(K_{0}\right)$ by the geodesic symmetry through $x_{0}$. In this case the only standard ends of $\mathbb{R}_{n-1}^{n}$ are the end of $\mathbb{R}_{n-1}^{n}$ and the end of the orbit space $\mathbb{Z}_{2} \backslash \mathbb{R}_{n-1}^{n}\left(K_{0}\right)$.

Definition 6.2. Let $K_{0}>0$ and $1 \leq k<n-1$ Let $G \subset O(n-k+1) \times O(k)$ be a weakly fixed point free finite group. Then the end of the quotient $G \backslash \mathbb{R}_{k}^{n}$ constructed above is a standard end. If $k=n-1$, then the standard end is as just described. If $K_{0}<0$ then the space $\mathbb{R}_{k}^{n}\left(K_{0}\right)$ is anti-isometric to the space $\mathbb{R}_{n-k}^{n}\left(-K_{0}\right)$. Thus we use the constructions just given to define a standard end in this case. 
Let $E$ be an end of the manifold $M$. Recall that the fundamental group $\pi_{1}(E)$ is isomorphic to $G$ iff for every compact set $C_{1} \subset M$ there is a compact set $C_{2} \supseteq C_{1}$ so that $\pi_{1}\left(E \backslash C_{2}\right.$ is isomorphic to $G$ and if $i:\left(E \backslash C_{2}\right) \rightarrow\left(E \backslash C_{1}\right)$ is inclusion, then the induced map $i_{*}: \pi_{1}\left(E \backslash C_{2}\right) \rightarrow \pi_{1}\left(E \backslash C_{1}\right)$ is injective. In particular this means the end $E$ is simply connected if of all compact $C_{1}$ there is a $C_{2} \supseteq C_{1}$ so that $E \backslash C_{2}$ is simply connected.

In some cases the model space $\mathbb{R}_{k}^{n}\left(K_{0}\right)$ will have two ends. In this case the two ends are isometric and so the isomorphism class of an end of $\mathbb{R}_{k}^{n}\left(K_{0}\right)$ is well defined.

Table 3.

\begin{tabular}{|c|c|c|c|c|c|c|}
\hline$K_{0}$ & $k$ & Diff. Type & Positive & Negative & \# of ends & $\pi_{1}(\infty)$ \\
\hline \hline$K_{0}=0$ & $1 \leq k \leq n-1$ & $\mathbb{R}^{n}$ & $\infty$ & $\infty$ & 1 & $\langle 0\rangle$ \\
\hline$K_{0}=0$ & $k=0$ & $\mathbb{R}^{n}$ & $\infty$ & - & 1 & $\langle 0\rangle$ \\
\hline$K_{0}=0$ & $k=n$ & $\mathbb{R}^{n}$ & - & $\infty$ & 1 & $\langle 0\rangle$ \\
\hline$K_{0}<0$ & $k=0$ & $\mathbb{R}^{n}$ & $\infty$ & - & 1 & $\langle 0\rangle$ \\
\hline$K_{0}<0$ & $k=1$ & $\mathbb{R}^{n}$ & $\infty$ & $\infty$ & 1 & $\langle 0\rangle$ \\
\hline$K_{0}<0$ & $2 \leq k=n-1$ & $S^{n-1} \times \mathbb{R}$ & $\infty$ & $2 \pi / \sqrt{K_{0}}$ & 2 & $\langle 0\rangle$ \\
\hline$K_{0}<0$ & $2 \leq k \leq n-3$ & $S^{k} \times \mathbb{R}^{n-k}$ & $\infty$ & $2 \pi / \sqrt{K_{0}}$ & 1 & $\langle 0\rangle$ \\
\hline$K_{0}<0$ & $2 \leq k=n-2$ & $S^{k} \times \mathbb{R}^{2}$ & $\infty$ & $2 \pi / \sqrt{K_{0}}$ & 1 & $\mathbb{Z}$ \\
\hline$K_{0}<0$ & $k=n$ & $S^{n}$ & - & $2 \pi / \sqrt{K_{0}}$ & 0 & - \\
\hline$K_{0}>0$ & $k=0$ & $S^{n}$ & $2 \pi / \sqrt{K_{0}}$ & - & 0 & - \\
\hline$K_{0}>0$ & $k=n$ & $\mathbb{R}^{n}$ & - & $\infty$ & 1 & $\langle 0\rangle$ \\
\hline$K_{0}>0$ & $k=n-1$ & $\mathbb{R}^{n}$ & $\infty$ & $\infty$ & 1 & $\langle 0\rangle$ \\
\hline$K_{0}>0$ & $k=1, n \geq 3$ & $S^{n-1} \times \mathbb{R}$ & $2 \pi / \sqrt{K_{0}}$ & $\infty$ & 2 & $\langle 0\rangle$ \\
\hline$K_{0}>0$ & $3 \leq k \leq n-2$ & $S^{n-k} \times \mathbb{R}^{k}$ & $2 \pi / \sqrt{K_{0}}$ & $\infty$ & 1 & $\langle 0\rangle$ \\
\hline$K_{0}>0$ & $2=k \leq n-2$ & $S^{n-k} \times \mathbb{R}^{2}$ & $2 \pi / \sqrt{K_{0}}$ & $\infty$ & 1 & $\mathbb{Z}$ \\
\hline
\end{tabular}

A more troublesome case is when the end of the model space is not simply connected. (This happens when $\mathbb{R}_{k}^{n}\left(K_{0}\right)$ is diffeomorphic to $\mathbb{R}^{2}$ or $S^{n-2} \times \mathbb{R}^{2}$ where $n \geq 4$.) These facts and some other information we will need is given in the following table. The column headed by "Diff. Type" gives the diffeomorphism type of $\mathbb{R}_{k}^{n}\left(K_{0}\right)$, the column headed by "Positive" (resp. "Negative") gives the lengths of the positive (resp. negative) geodesics (when this number is finite it means that all geodesics are closed with the given length as smallest period). The other two columns give the number of ends and the fundamental group of an end.

We will use the fact that when a geodesic has infinite length then it will eventually leave every compact set. Null geodesics have this property in all 
cases.

Theorem 6.3. Let $(M, g)$ be a geodesically complete semi-Riemannian manifold of dimension $n$ and index $k$ and let $E$ be an end of $M$. Assume that $E$ has finite fundamental group and constant sectional curvature $K_{0}$. Then the model space $\mathbb{R}_{k}^{n}\left(K_{0}\right)$ is non-compact, its ends are simply connected, and the end $E$ is isometric to a standard end of constant sectional curvature $K_{0}$ and index $k$.

This implies that when the model space $\left(\mathbb{R}_{k}^{n}\left(K_{0}\right), g_{\mathbb{R}_{k}^{n}\left(K_{0}\right)}\right)$ is diffeomorphic to $\mathbb{R}^{2}$ or $S^{n-2} \times \mathbb{R}^{2}$ for $n \geq 4$ (the cases where the end of $\mathbb{R}_{k}^{n}\left(K_{0}\right)$ has infinite fundamental group) then no end with a finite fundamental group can be geodesically complete and be locally isometric to an end of $\left(\mathbb{R}_{k}^{n}\left(K_{0}\right), g_{\mathbb{R}_{k}^{n}\left(K_{0}\right)}\right)$.

The proof of Theorem 6.3 breaks up into two parts. The first is understanding the simply connected ends of constant curvature and the second is in proving a fixed point theorem for finite groups of isometries on the model space. The facts about simply connected ends are given by

Proposition 6.4. Let $(M, g)$ be a geodesically complete semi-Riemannian manifold of dimension $n$ and index $k$. Assume that $M$ has non-empty compact boundary $\partial M$ and that $(M, g)$ is geodesically complete in the sense that every inextendible geodesic either has no endpoint or an endpoint on $\partial M$.

Assume that $(M, g)$ is simply connected and that it has constant sectional curvature $K_{0}$ and that the model space $\mathbb{R}_{k}^{n}\left(K_{0}\right)$ has simply connected ends. Then $k$ and $K_{0}$ are such that $\mathbb{R}_{k}^{n}\left(K_{0}\right)$ is noncompact and there are compact sets $\partial M \subset C_{1} \subset M$ and $C_{2} \subset \mathbb{R}_{k}^{n}\left(K_{0}\right)$ so that every unbounded component of $M \backslash C_{1}$ is isometric with an unbounded component of $\mathbb{R}_{k}^{n}\left(K_{0}\right) \backslash C_{2}$.

Proof. As $M$ is simply connected and locally isomorphic to $\mathbb{R}_{k}^{n}\left(K_{0}\right)$ a standard monodromy argument shows there is a local isometry $f: M \rightarrow \mathbb{R}_{k}^{n}\left(K_{0}\right)$. Let $U$ be a connected component of $\mathbb{R}_{k}^{n}\left(K_{0}\right) \backslash f[\partial M]$ and set $\widehat{U}:=f^{-1}[U]$. If $\widehat{U} \neq \emptyset$ then $\left.f\right|_{\mathbb{U}}: \widehat{U} \rightarrow U$ is a covering map. To see this let $y \in U$ and let $B \subset U$ be a geodesically convex neighborhood of $y$ whose closure is disjoint from $f[\partial M]$ and let $N_{y} \subset T\left(\mathbb{R}_{k}^{n}\left(K_{0}\right)\right)_{y}$ be the convex neighborhood of the origin so that $\exp \left[N_{y}\right]=B_{y}$. Let $x \in \widehat{U}$ so that $f(x)=y$. Let $\widehat{N}_{x} \subset T(M)_{x}$ be the convex neighborhood of the origin so that $f_{* x}\left[\widehat{N}_{x}\right]=N_{y}$ and let $\widehat{B}_{x}=\exp \left[\widehat{N}_{x}\right]$. Then $\widehat{B}_{x} \subset \widehat{U}$, and $\left.f\right|_{B_{x}}: \widehat{B}_{x} \rightarrow B_{x}$ is a diffeomorphism. As 
$x$ was any point of $\widehat{U}$ with $f(x)=y$ this shows that the neighborhood $B_{x}$ is evenly covered. Thus if $\widehat{U} \neq \emptyset$ the map $\left.f\right|_{0}$ is a covering map as claimed.

Claim. The set $f[M]$ is not contained in an compact subset of $\mathbb{R}_{k}^{n}\left(K_{0}\right)$.

Assuming the claim we prove the proposition. Let $U$ be an unbounded component of $\mathbb{R}_{k}^{n}\left(K_{0}\right) \backslash f[\partial M]$ so that $f[M] \cap U \neq \emptyset$ and set $\widehat{U}:=f^{-1}[U]$. Then $\left.f\right|_{U}: \widehat{U} \rightarrow U$ is a covering map. Then there is a compact set $C_{2} \supset$ $f[\partial M]$ so that $U_{2}:=U \backslash C_{2}$ is simply connected. Let $\widehat{U}_{2}$ be a connected component of $f^{-1}\left[U_{2}\right]$. As $U_{2}$ is simply connected the map $\left.f\right|_{\hat{U}_{2}}: \widehat{U}_{2} \rightarrow U_{2}$ must be a diffeomorphism and thus an isometry. As $\partial U_{2}$ is compact the boundary of $\widehat{U}_{2}$ is also compact. The proposition now follows.

We now prove the claim. This is complicated by the fact that in many cases the model space $\mathbb{R}_{k}^{n}\left(K_{0}\right)$ contains closed geodesics. This means it might be possible to have a geodesic ray $\gamma:[0, \infty) \rightarrow M$ that eventually leaves every compact set of $M$, but $f \circ \gamma:[0, \infty) \rightarrow \mathbb{R}_{k}^{n}\left(K_{0}\right)$ is closed and thus is contained in a compact subset of $\mathbb{R}_{k}^{n}\left(K_{0}\right)$. To avoid this note that by multiplying the metric of $\mathbb{R}_{k}^{n}\left(K_{0}\right)$ by \pm 1 we can assume that no non-negative geodesic of $\mathbb{R}_{k}^{n}\left(K_{0}\right)$ is closed and that in fact a non-negative geodesic eventually leaves every compact set.

Let $D$ be a compact subdomain of $M$ with smooth boundary and so that $\partial M$ is a subset of the interior of $D$. We also choose a complete Riemannian metric $h$ on $M$ (which has no relation to the semi-Riemannian metric $g$ on $M$ ). Let $S^{+}(D)$ be the set of all non-negative (i.e. $\left.g(u, u) \geq 0\right) h$ unit vectors tangent to $M$ at a point of $D$. Then all the fibers of $S^{+}(D)$ are compact and $D$ is compact so the space $S^{+}(D)$ is compact. Let $V$ be an unbounded component of $M \backslash D$ and let $\mathcal{G}$ be the compact set of ordered pairs $(u, t) \in S^{+}(D) \times(0, \infty)$ so that $\exp (t u) \in V$ and $0 \leq t \leq 1$. (Here exp is the exponential of the semi-Riemannian metric $g$.) Note that if $x \in \partial D \cap \partial V$ then there is a positive $h$-unit vector $u$ at $x$ pointing into $V$ and thus $\exp (t u) \in V$ for small $t$ and thus $\mathcal{G}$ is not empty. For each $(u, t) \in \mathcal{G}$ let $(\alpha(u, t), \beta(u, t))$ be the maximal interval containing $t$ and contained in the set $\{s: \exp (s u) \in V\}$. Thus $0 \leq \alpha(u, t)<t<\beta(u, t) \leq \infty$ and $\alpha(u, t) \leq 1$. If for some $(u, t) \in \mathcal{G}$ we have $\beta(u, t)=\infty$ then $\gamma(s)=f(\exp (s u))$ with $s>\alpha(u, t)$ is a non-negative geodesic ray of $\mathbb{R}_{k}^{n}\left(K_{0}\right)$ and thus it eventually leaves every compact set. Thus $f[M]$ is not contained in any compact subset of $\mathbb{R}_{k}^{n}\left(K_{0}\right)$ and thus the claim holds in this case.

Next assume that there is a sequence $\left\{\left(u_{l}, t_{l}\right)\right\}_{l=1}^{\infty} \subset \mathcal{G}$ so that

$$
\lim _{l \rightarrow \infty} \beta\left(u_{l}, t_{l}\right)=\infty
$$


Then as $t_{l} \in[0,1]$ and $S^{+}(D)$ is compact, by going to a subsequence we can assume $t_{l} \rightarrow t_{0}$ for some $t_{0} \in[0,1]$ and $u_{l} \rightarrow u_{0}$ for some $u_{0} \in S^{+}(D)$. Consider the geodesic

$$
\gamma(s)=\exp \left(s u_{0}\right)=\lim _{l \rightarrow \infty} \exp \left(s u_{l}\right)
$$

for $s>1$. This will be disjoint from $D^{\circ}$ (the interior of $D$ ) and thus does not meet $\partial M$. It is also a non-negative geodesic. Thus the geodesic $f(\gamma(s))$ in $\mathbb{R}_{k}^{n}\left(K_{0}\right)$ will eventually leave every compact set and thus $f[M]$ is not contained in any compact subset of $\mathbb{R}_{k}^{n}\left(K_{0}\right)$.

This leaves the case where the function $\beta(u, t)$ is bounded on $\mathcal{G}$, say $\beta(u, t) \leq C_{0}$. Then let

$$
G=\{\exp (s u): \alpha(u, t)<s<\beta(u, t) \quad \text { for some } \quad(u, t) \in \mathcal{G}\} .
$$

Consider the sequence $\left\{\exp \left(s_{l} u_{l}\right)\right\}_{l=1}^{\infty} \subset G$ where $\left(u_{l}, t_{l}\right)$ is a sequence in $\mathcal{G}$. By passing to a subsequence we can assume that $t_{l} \rightarrow t_{0}$ and $u_{l} \rightarrow u_{0}$ for some $u_{0} \in S^{+}(D)$. As $s_{l}<\beta\left(u_{l}, t_{l}\right) \leq C_{0}$ we can also assume $s_{l} \rightarrow s_{0}$ for some $s_{0} \leq C_{0}$. Then $\exp \left(s_{l} u_{l}\right) \rightarrow \exp \left(s_{0} u_{0}\right)$ which shows that every sequence out of $G$ has a convergent subsequence. Therefore so the closure of $G$ is compact in $M$. As the component $V$ is unbounded it can not be contained in any compact set and thus there is a point $x$ in $V$ that is not in $G$. Let $\gamma$ be a positive geodesic of $M$ through the point $x$. Then $\gamma$ can not meet $D$, for if it did the point $x$ would be of the form $\exp (s u)$ for some $(u, t) \in \mathcal{G}$ contradicting that $x$ is not in $G$. Thus the geodesic $f \circ \gamma$ of $\mathbb{R}_{k}^{n}\left(K_{0}\right)$ leaves every compact set and completes the proof of the claim $f[M]$ is not contained in any compact subset of $\mathbb{R}_{k}^{n}\left(K_{0}\right)$. This also completes the proof of the proposition.

To pass from the case of simply connected ends to the case of ends with finite fundamental group we need information about finite group actions on certain homogeneous spaces of totally geodesic submanifolds of $\mathbb{R}_{k}^{n}\left(K_{0}\right)$. By a maximal compact totally geodesic submanifold in $\mathbb{R}_{k}^{n}\left(K_{0}\right)$ we mean a compact totally geodesic submanifold $C$ of $\mathbb{R}_{k}^{n}\left(K_{0}\right)$ which is not properly contained in any other compact totally geodesic submanifold of $\mathbb{R}_{k}^{n}\left(K_{0}\right)$. In many cases the maximal compact totally geodesic submanifolds may just be points. In the case the space $\mathbb{R}_{k}^{n}\left(K_{0}\right)$ is compact, then the only maximal compact flat is $\mathbb{R}_{k}^{n}\left(K_{0}\right)$ itself. The following gives the basic facts. The proof is left to the reader.

Proposition 6.5. If $C_{1}$ and $C_{2}$ are maximal compact totally geodesic submanifolds in $\mathbb{R}_{k}^{n}\left(K_{0}\right)$, then there is an isometry $g$ of $\mathbb{R}_{k}^{n}\left(K_{0}\right)$ with $g C_{1}=C_{2}$. 
Thus all the maximal compact totally geodesic submanifolds in $\mathbb{R}_{k}^{n}\left(K_{0}\right)$ are isometric. The maximal compact totally geodesic submanifolds are

Table 4.

\begin{tabular}{|c|c|c|}
\hline$K_{0}$ & $n, k$ & Max. Cpt. Tot. Geo. Subman. \\
\hline \hline$K_{0}=0$ & $0 \leq k \leq n$ & Point \\
\hline$K_{0}>0$ & $k=n-1, n$ & Point \\
\hline$K_{0}>0$ & $0 \leq k \leq n-2$ & $S^{n-k}\left(1 / \sqrt{K_{0}}\right)$ \\
\hline$K_{0}<0$ & $k=0,1$ & Point \\
\hline$K_{0}<0$ & $2 \leq k \leq n$ & $S^{k}\left(1 / \sqrt{-K_{0}}\right)$ \\
\hline
\end{tabular}

For the rest of this section we will denote the space of maximal compact totally geodesic submanifolds in $\mathbb{R}_{k}^{n}\left(K_{0}\right)$ by $\operatorname{MCTGS}\left(n, k, K_{0}\right)$. Note that if the elements of $\operatorname{MCTGS}\left(n, k, K_{0}\right)$ are points, then $\operatorname{MCTGS}\left(n, k, K_{0}\right)=$ $\mathbb{R}_{k}^{n}\left(K_{0}\right)$.

Proposition 6.6. $K_{0}>0$ and $k \neq n-1$ or $K_{0}<0$ and $k \neq 1$ then $\operatorname{MCTGS}\left(n, k, K_{0}\right)$ can be given the structure of a Riemannian symmetric space of non-compact type in such a way that the group of isometries of $\mathbb{R}_{k}^{n}\left(K_{0}\right)$ acts on $\operatorname{MCTGS}\left(n, k, K_{0}\right)$ by isometries. In particular the sectional curvature on $\operatorname{MCTGS}\left(n, k, K_{0}\right)$ is non-positive.

Proof. As the spaces $\mathbb{R}_{k}^{n}\left(K_{0}\right)$ and $\mathbb{R}_{n-k}^{n}\left(-K_{0}\right)$ are anti-isomorphic, it is enough to prove the result in the case $K_{0}>0$. When $K_{0}>0$ the full isometry group of $\mathbb{R}_{k}^{n}\left(K_{0}\right)$ is the orthogonal group $O(n-k+1, k)$. If $C_{0} \in$ MCTGS then the subgroup of $O(n-k+1, k)$ fixing $C_{0}$ is $O(n-k+1) \times O(k)$ and thus as a homogeneous space MCTGS $=O(n-k+1, k) /(O(n-$ $k+1) \times O(k))$. This is well known to be a Riemannian symmetric space ([20, p518] it is the non-compact dual to the Grassmannian manifold $\left.G_{k}\left(\mathbb{R}^{n+1}\right)=O(n) /(O(n-k+1) \times O(k))\right)$. It is a standard result that MCTGS $=O(n-k+1, k) /(O(n-k+1) \times O(k))$ has non-positive curvature as it is a Riemannian symmetric space of non-compact type. Cf. [20, Theorem 3.1 p. 241].

Proposition 6.7. Let $H$ be a compact group acting on $\mathbb{R}_{k}^{n}\left(K_{0}\right)$ by isometries. Then $H$ fixes some point of $\operatorname{MCTGS}\left(n, k, K_{0}\right)$. 
Proof. If $K_{0}=0$ then $H$ is a compact group acting on $\mathbb{R}^{n}=\mathbb{R}_{k}^{n}(0)$ by affine maps. Thus $H$ must fix a point of $\mathbb{R}_{k}^{n}\left(K_{0}\right)$ which proves the result in this case as the maximal compact totally geodesic submanifolds are points.

If $K_{0}>0$ and $k \neq n-1$ of $K_{0}<0$ and $k \neq 1$ then by the last proposition $\operatorname{MCTGS}\left(n, k, K_{0}\right)$ is a simply connected Riemannian manifold with non-positive sectional curvature and $H$ acts on $\operatorname{MCTGS}\left(n, k, K_{0}\right)$ by isometries. So by the Cartan fixed point theorem [20, Theorem 13.5, p. 75] $H$ fixes a point of $\operatorname{MCTGS}\left(n, k, K_{0}\right)$.

This leaves the equivalent cases of $K_{0}>0$ and $k=n-1$ and $K_{0}<0$ and $k=1$. We will deal with the case where $K_{0}<0$ and $k=1$ and we normalize so that $K_{0}=-1$. Let $\mathbb{R}_{2}^{n+1}$ be $\mathbb{R}^{n+1}$ with the inner product

$$
\langle x, y\rangle=-x_{1} y_{1}-x_{2} y_{2}+x_{3} y_{3}+\cdots+x_{n+1} y_{n+1} .
$$

Let $S_{1}^{n}(-1)$ be the hypersurface in $\mathbb{R}_{2}^{n+1}$ defined by $\langle x, x\rangle=-1$. Then $S_{1}^{n}(-1)$ has constant sectional curvature -1 and is diffeomorphic to $S^{1} \times$ $\mathbb{R}^{n-1}$.

Let $p: \mathbb{R}_{1}^{n}(-1) \rightarrow S_{1}^{n}(-1)$ be the covering map and let $\Gamma$ be the group of deck transformations. Recall that $\mathbb{R}_{1}^{n}(-1)$ is time orientable. Fix a time orientation on $\mathbb{R}_{1}^{n}(-1)$. Because each timelike geodesic of $S_{1}^{n}(-1)$ is closed of length $2 \pi$ it is not hard to see that the group $\Gamma$ of deck transformations is cyclic and its generator is the unique isometry $a$ of $\mathbb{R}_{1}^{n}(-1)$ with the property that for every future pointing timelike unit speed geodesic $\gamma$ in $\mathbb{R}_{1}^{n}(-1)$ there holds $a \gamma(t)=\gamma(t+2 \pi)$.

Let $g$ be any isometry of $\mathbb{R}_{1}^{n}(-1)$ that preserves the time orientation of $\mathbb{R}_{1}^{n}(-1)$. Then for any future pointing time like unit speed geodesic $\gamma$ the curve $g \gamma$ is also a future pointing time like unit speed geodesic and so $a g \gamma(t)=g \gamma(t+2 \pi)=g a \gamma(t)$. As there is a future pointing time like unit speed geodesic through any point of $\mathbb{R}_{1}^{n}(-1)$ this implies $a g=g a$. That is the generator $a$ of $\Gamma$ commutes with every isometry of $\mathbb{R}_{1}^{n}(-1)$ that preserves the time orientation.

We now prove the proposition in the special case where every element of the group $H$ preserves the time orientation of $\mathbb{R}_{1}^{n}$. Then every element of $H$ commutes with every element of the group of deck transformations and therefore $H$ also acts on $S_{1}^{n}(-1)$ by $g p(x):=p(g x)$. We now claim that there is a time like geodesic of $S_{1}^{n}(-1)$ that is fixed by the action of $H$. Note that the group of isometries of $S_{1}^{n}(-1)$ that stabilize some fixed timelike geodesic is $O(2) \times O(n-1)$ and the full isometry group of $S_{1}^{n}(-1)$ is $O(2, n-1)$. Therefore as a homogeneous space, the space of timelike geodesics of $S_{1}^{n}(-1)$ is $O(2, n-1) /(O(2) \times O(n-1))$ which, just as above, is 
a Riemannian symmetric space with non-positive sectional curvature. Thus the Cartan fixed point theorem lets us conclude $H$ fixes a timelike geodesic $c$ of $S_{1}^{n}(-1)$.

Let $\gamma$ be the future pointing unit speed time like geodesic covering of $S_{1}^{n}(-1)$ covering $c$. Then $\gamma$ is fixed set-wise by every element $g \in H$. As the elements of $H$ preserve the time orientation this implies that if $g \in H$, then $g \gamma(t)=\gamma\left(t+t_{0}\right)$ for some $t_{0} \in \mathbb{R}^{n}$. But then $g^{l} \gamma(t)=\gamma\left(t+l t_{0}\right)$. But as the group $H$ is compact the orbit $\left\{g^{l} \gamma(0)=\gamma\left(l t_{0}\right): l=0, \pm 1, \pm 2, \ldots\right\}$ must have compact closure in $\mathbb{R}_{1}^{n}(-1)$ which is only the case if $t_{0}=0$. Thus $g \gamma(t)=\gamma(t)$ for all $g \in H$. So in the case where all elements of $H$ preserve the time orientation of $\mathbb{R}_{1}^{n}(-1)$ there is always a time like geodesic that is fixed pointwise by $H$.

Finally we consider the case where the group contains elements that reverse the time orientation of $\mathbb{R}_{1}^{n}(-1)$. In this case let $H_{0}$ be the subgroup of $H$ of elements that preserve the the time orientation of $\mathbb{R}_{1}^{n}(-1)$. Then $H_{0}$ is a subgroup of $H$ of index 2. We consider two cases. First assume every element of $H_{0}$ fixes every element of $\mathbb{R}_{1}^{n}(-1)$. Then $H_{0}=\langle 1\rangle$ and so $H$ is just a cyclic group of order two. Let $b$ be the generator of $H$. Then $b$ reverses the time orientation of $\mathbb{R}_{1}^{n}(-1)$. Choose a fixed future pointing time like unit speed geodesic $\gamma_{0}$ for reference and let $\tau$ be the time function determined by $\gamma_{0}$, cf. Definition 2.8. That is, on $\gamma_{0}$ the function is given by $\tau\left(\gamma_{0}(t)\right)=t$ and then $\tau$ is extended to $\mathbb{R}_{1}^{n}(-1)$ to be constant on the totally geodesic hypersurfaces perpendicular to $\gamma_{0}$. As the element $b$ of $H$ reverses the time orientation of $\mathbb{R}_{1}^{n}(-1)$ the function $\tau \circ b$ is a decreasing function when restricted to $\gamma_{0}, \lim _{t \rightarrow \infty} \tau(b \gamma(t))=-\infty$, and $\lim _{t \rightarrow-\infty} \tau(b \gamma(t))=+\infty$. Thus there is a point $x=\gamma\left(t_{1}\right)$ so that $\tau(x)=\tau(g x)$.

Let $M$ be the set of all points of $\mathbb{R}_{1}^{n}(-1)$ where $\tau(x)=\tau(b x)$. Then $M$ is not empty and as the level sets of both $\tau$ and $\tau \circ b$ are totally geodesic spacelike hypersurfaces and $M$ is the intersection of two such level sets, $M$ will be a space like totally geodesic submanifold of either codimension 1 (in the case the level sets happen to be equal) or codimension 2 (in all other cases). This implies $M$ is a simply connected Riemannian manifold of constant non-positive sectional curvature. As the isometry $b$ leaves $M$ fixed set-wise, the Cartan fixed point theorem can again be used to show $b$ has a fixed point on $M$. This completes the proof in the case $H_{0}=\langle 1\rangle$.

This leaves the case where $H_{0} \neq\langle 1\rangle$. Then by what we have shown above there is a timelike geodesic $\gamma$ that is fixed pointwise by $H_{0}$. Let $N$ be the subset of $\mathbb{R}_{1}^{n}(-1)$ of all points fixed by all elements of $H_{0}$. As $N$ is the fixed point set of a group of isometries it is a totally geodesic submanifold of $\mathbb{R}_{1}^{n}(-1)$. As $N$ contains a timelike geodesic the restriction of the metric to 
$N$ is non-degenerate (in Lorentzian manifolds the only degenerate subspaces have a positive semidefinite metric). As $H_{0} \neq\langle 1\rangle$ the submanifold $N$ has dimension less than $n$. As the subgroup $H_{0}$ of $H$ has index two it is normal in $H$. This implies that the set $N$ is fixed set-wise by the group $H$. To see this let $a \in H_{0}$ and $g \in H$. Then $g^{-1} a g \in H_{0}$ and so for $x \in N$ we have $a g x=g g^{-1} a g x=g x$. Thus $g x$ is fixed by all elements of $H_{0}$ and so $g x \in N$ as claimed. Thus $H$ acts on the submanifold $N$ and $N$ is isometric to $\mathbb{R}_{1}^{m}(-1)$ for some $m<n$. Thus we can now use induction to conclude that $H$ has a fixed point in $N$ and therefore also in $\mathbb{R}_{1}^{n}(-1)$. This completes the proof.

Proof of Theorem 6.3. Let $E$ be an end of $M$ with constant sectional curvature and finite fundamental group $\pi_{1}(E)$. Then there is a compact set $C$, which we can assume to have smooth boundary, so that $E \backslash C$ has fundamental group isomorphic to $\pi_{1}(E)$. Let $\pi: N \rightarrow E \backslash C$ be the universal cover of $E \backslash C$ and let $G$ be the group of deck transformations of this cover. Thus $G$ acts on the semi-Riemannian manifold $N$ by isometries and $G$ and the orbit space $G \backslash N$ is isometric to $E \backslash C$. Thus it is enough to show $G \backslash N$ is isometric to a standard end. By use of Proposition 6.4 by replacing $C$ by a larger compact set we can assume $N$ is isometric to an unbounded component of $\mathbb{R}_{k}^{n}\left(K_{0}\right) \backslash C_{2}$ for some compact $C_{2}$. Thus we simply assume $N=\mathbb{R}_{k}^{n}\left(K_{0}\right) \backslash C_{2}$. As the space $\mathbb{R}_{k}^{n}\left(K_{0}\right)$ is simply connected, the action of $G$ on $N$ extends to an action on the whole space $\mathbb{R}_{k}^{n}\left(K_{0}\right)$. By Proposition 6.7 the group fixes some element of $\operatorname{MCTGS}\left(n, k, K_{0}\right)$. If the maximal compact totally geodesic submanifolds of $\mathbb{R}_{k}^{n}\left(K_{0}\right)$ are spheres $S^{p}$ with $p \geq 2$, then by normalizing the metric we can assume the space is $\left(S^{p} \times \mathbf{H}^{n-p}, \cosh ^{2}(\rho) g_{\mathbf{S}}-\right.$ $\left.g_{\mathbf{H}}\right)$ and that the fixed element of $\operatorname{MCTGS}\left(n, k, K_{0}\right)$ is $S^{p} \times\{\rho=0\}$. Then it follows that $G$ must be fixed weakly fixed point free and that $G \backslash N$ is a standard end.

If the maximal compact totally geodesic submanifolds are points, then let $x_{0}$ be the point of $\mathbb{R}_{k}^{n}\left(K_{0}\right)$ fixed by $G$. Then there is a $k$-dimensional subspace $V$ of $T\left(\mathbb{R}_{k}^{n}\left(K_{0}\right)\right)_{x_{0}}$ that is fixed by $G$. (I.e. $a_{* x_{0}} V=V$ for all $a \in G$.) This is because the set of all such subspaces is just the homogeneous space $O(k, n-k) /(O(k) \times O(n-k)$ and as we have done several times above, we can apply the Cartan fixed point theorem to this case. But then by standard linear algebra the orthogonal complement $V^{\perp}$ of $V$ is also invariant under $G$. Letting $S^{k-1} \times S^{n-k-1}$ be the product of the unit spheres in these spaces we see that the group has an action on $S^{k-1} \times S^{n-k-1}$ which must then be strongly fixed point free and thus the space $G \backslash N$ is again isomorphic 
to a standard end. This completes the proof.

\subsubsection{Rigidity Results.}

Theorem 6.8. Let $(\bar{M}, \bar{g})$ be a geodesically complete semi-Riemannian manifold of dimension $n \geq 3$ and index $k$ with curvature satisfying one of the two inequalities $\bar{R} \geq 0$ or $\bar{R} \leq 0$ and assume $(\bar{M}, \bar{g})$ has an end $E$ with $\bar{R} \equiv 0$ on $E$ and $\pi_{1}(E)$ finite. Then $(\bar{M}, \bar{g})$ is isometric to the flat model space $\left(\mathbb{R}_{k}^{n}, g_{0}\right)$.

Theorem 6.9. Let $(\bar{M}, \bar{g})$ be a geodesically complete Lorentzian or Riemannian manifold of dimension $n \geq 3$ with curvature tensor satisfying one of the two one sided inequalities $\bar{R} \geq-1$ or $\bar{R} \leq-1$. Assume $(\bar{M}, \bar{g})$ has an end $E$ with $\pi_{1}(E)$ finite and so that $\bar{R} \equiv-1$ on $E$. Then if $(\bar{M}, \bar{g})$ is Lorentzian it is isometric to the anti-de Sitter space $\left(\mathbb{R}_{1}^{n}(-1), g_{\mathbb{R}_{1}^{n}(-1)}\right)$. If $(\bar{M}, \bar{g})$ is Riemannian it is isometric to the hyperbolic space $\left(\mathbb{R}_{0}^{n}(-1), g_{\mathbb{R}_{0}^{n}(-1)}\right)$

Proofs. We first consider Theorem 6.8. By Theorem 6.3 there is a compact set $C \subset \mathbb{R}_{k}^{n}$ and an local isometry $\varphi: \mathbb{R}_{k}^{n} \backslash C \rightarrow \bar{M}$ so that for each $y \in \bar{M}$ the preimage $\varphi^{-1}[y]$ has at most $\#\left(\pi_{1}(E)\right)$ elements. By Theorem 4.2 this extends to a surjective local isometry $\widehat{\varphi}: \mathbb{R}_{k}^{n} \rightarrow \bar{M}$. This implies the map $\widehat{\varphi}$ is a covering map and that the group of deck transformations is isomorphic to $G:=\pi_{1}(E)$ and thus $G$ is finite. By Proposition $6.7 G$ has a fixed point on $\mathbb{R}_{k}^{n}$. But as $G$ is the group of deck transformations of $\widehat{\varphi}: \mathbb{R}_{k}^{n} \rightarrow M$ the only way it can have a fixed point is if it is the trivial group $G=\langle 1\rangle$. This implies $\hat{\varphi}$ is an isometry and completes the proof of Theorem 6.8. The proof of Theorem 6.9 is identical except that the rigidity results of Section 5.2 are used.

Remark 6.10. In all cases not covered by the last two theorems the exact analog of the theorems is false. First consider the case where $K_{0}<0$ and $3 \leq$ $k \leq n-1$. Normalize so that $K_{0}=-1$. Then as above $\mathbb{R}_{k}^{n}\left(K_{0}\right)$ is isometric to the warped product metric $g_{0}=-\cosh ^{2}(\rho) g_{\mathbf{S}}+g_{\mathbf{H}}$ on $S^{k} \times \mathbf{H}^{n-k}$. Thus if $G$ is a finite group of isometries of $\left(S^{k}, g_{\mathbf{S}}\right)$ acting without fixed points, then it also has a fixed point free action on $\mathbb{R}_{k}^{n}\left(K_{0}\right) \approx S^{k} \times \mathbf{H}^{n-k}$ by letting it act on the first factor. Then the manifold $G \backslash \mathbb{R}_{k}^{n}\left(K_{0}\right)$ has a metric of constant sectional curvature -1 , and an end with a finite fundamental group without being isometric to $\mathbb{R}_{k}^{n}\left(K_{0}\right)$. There are similar examples when $K_{0}>0$. 
However it is still reasonable to conjecture that if $(M, g)$ is a geodesically complete semi-Riemannian manifold of dimension $n \geq 3$ and index $k$ with $3 \leq k \leq n-1$, which satisfies an appropriate one sided bound on sectional curvature and has an end $G$ with finite fundamental group and constant sectional curvature $K_{0}<0$ that $(M, g)$ is isometric to a quotient of $\mathbb{R}_{k}^{n}\left(K_{0}\right)$ by a finite fixed point free group of isometries. Getting control of the geometry of the end is taken care of by Theorem 6.3. What is needed to prove this is an extension of the rigidity results of Section 5.2 to the case of space forms of index other than $k=0,1, n-1, n$.

\subsection{Rigidity in Quotients.}

Definition 6.11. Let $M$ be a smooth manifold and let $C \subset M$ be a closed subset. Let $g_{1}$ and $g_{2}$ be two smooth semi-Riemannian metrics on $M$ and let $\ell$ be a non-negative integer. Then we say that $g_{1}$ and $g_{2}$ agree to order $\ell$ on $C$ if and only if for any point $x \in C$ the partial derivatives of $g_{1}$ and $g_{2}$ of order at most $\ell$ are equal in any local coordinate system containing $x$.

Remark 6.12. If $C$ is the closure of an open set and $g_{1}=g_{2}$ on $C$, then $g_{1}$ and $g_{2}$ agree to order $\ell$ on $C$ for all $\ell$.

Theorem 6.13. Let $\left(\bar{M}, \bar{g}_{0}\right)$ be a geodesically complete semi-Riemannian manifold of dimension at least three with constant curvature 0 . Let $U \subset \bar{M}$ be a connected open set with compact closure and assume that $\pi_{1}(U)$ is finite. Further, let $\bar{g}$ be a semi-Riemannian metric on $\bar{M}$ and assume that $\bar{g}=\bar{g}_{0}$ to order 2 on $\bar{M} \backslash U$ and that the curvature tensor $\bar{R}$ of $\bar{M}$ satisfies a one sided curvature bound

$$
\bar{R} \geq 0 \quad \text { or } \quad \bar{R} \leq 0 .
$$

Then $(\bar{M}, \bar{g})$ is isometric to $\left(\bar{M}, \bar{g}_{0}\right)$ and thus $(\bar{M}, \bar{g})$ has constant sectional curvature 0 .

Theorem 6.14. Let $\left(\bar{M}, \bar{g}_{0}\right)$ be a geodesically complete Riemannian or Lorentzian manifold of dimension at least three which is locally isometric to $\left(\mathbb{R}_{k}^{n}(-1), g_{\mathbb{R}_{k}^{n}(-1)}\right)$. (Thus $k=0$ (Riemannian) or $k=1$ (Lorentzian).) Let $U \subset \bar{M}$ be a connected open set with compact closure and assume $\pi_{1}(U)$ is finite. Further let $\bar{g}$ be a semi-Riemannian metric on $\bar{M}$ that agrees with 
$\bar{g}_{0}$ to order 2 on $\bar{M} \backslash U$ and so that the curvature of $\bar{g}$ satisfies a one sided curvature bound

$$
\bar{R} \geq-1, \quad \text { or } \quad \bar{R} \leq-1 .
$$

Then $(\bar{M}, \bar{g})$ is isometric to $\left(\bar{M}, \bar{g}_{0}\right)$.

Remark 1. If the manifold $\bar{M}$ is compact, then it is possible that the open set $U$ of the theorems is dense in $\bar{M}$. We look at an example of this in the Riemannian case. Let $\left(\bar{M}, \bar{g}_{0}\right)$ be a compact Riemannian manifold of constant sectional curvature $K_{0} \leq 0$. Then the fundamental group $\pi_{1}(\bar{M})$ is infinite. Let $x \in \bar{M}$ and let $C(x)$ be the cut locus of $x$ in $\bar{M}$. Then $U:=\bar{M} \backslash C(x)$ is simply connected (as it is homeomorphic to a ball in $\mathbb{R}^{n}$ ). Thus the theorems say that any Riemannian metric $\bar{g}$ on $\bar{M}$ that agrees with $\bar{g}_{0}$ to order 2 on $C(x)$ and satisfies a one sided curvature bound of the form (6.1) or (6.2), then $\bar{g}$ also has constant sectional curvature.

Proof of the Theorems. We prove Theorem 6.14, the proof of Theorem 6.13 being similar. The universal covering space of $\bar{M}$ is $\mathbb{R}_{k}^{n}(-1)$. Let $\pi: \mathbb{R}_{k}^{n}(-1) \rightarrow \bar{M}$ be the covering map. Then $\pi:\left(\mathbb{R}_{k}^{n}(-1), g_{\mathbb{R}_{k}^{n}(-1)}\right) \rightarrow$ $\left(\bar{M}, \bar{g}_{0}\right)$ is a local isometry. Let $\widehat{U}_{0}$ be a connected component of $\pi^{-1}[U]$. As $\pi_{1}(U)$ is finite, the map $\left.\pi\right|_{0_{0}}: \widehat{U}_{0} \rightarrow U$ is a finite sheeted cover. But then using that the closure of $U$ in $\bar{M}$ is compact, it is not hard to see that the closure of $\widehat{U}_{0}$ in $\mathbb{R}_{k}^{n}(-1)$ is compact. Define a new semi-Riemannian metric $g$ on $\mathbb{R}_{k}^{n}(-1)$ by

$$
g=\left\{\begin{array}{rll}
\pi^{*} \bar{g} & \text { on } & \widehat{U}_{0} \\
\pi^{*} \bar{g}_{0}=g_{\mathbb{R}_{k}^{n}(-1)} & \text { on } & \mathbb{R}_{k}^{n}(-1) \backslash \widehat{U}_{0} .
\end{array}\right.
$$

As $\pi:\left(\mathbb{R}_{k}^{n}(-1), g_{\mathbb{R}_{k}^{n}(-1)}\right) \rightarrow\left(\bar{M}, \bar{g}_{0}\right)$ is a local isometry and $\bar{g}$ and $\bar{g}_{0}$ agree to order 2 on $\partial U$ the semi-Riemannian metric $g$ pieces together to form a $C^{2}$ metric on $\mathbb{R}_{k}^{n}(-1)$. This semi-Riemannian metric agrees with the standard metric $g_{\mathbb{R}_{k}^{n}(-1)}$ outside of the compact set closure $\left(\widehat{U}_{0}\right)$.

It is easily checked that the proof of the rigidity result Theorem 5.8 goes through under the assumption the metrics are $C^{2}$. Therefore, we are able to conclude that $\left(\mathbb{R}_{k}^{n}(-1), g\right)$ is isometric to $\left(\mathbb{R}_{k}^{n}(-1), g_{\mathbb{R}_{k}^{n}(-1)}\right)$. Theorem 6.14 now follows.

The proof of Theorem 6.13 works along the same lines except that Corollary 4.4 is used to finish the proof. 


\section{References.}

[1] W. Ballmann, M. Gromov, and V. Schroeder, Manifolds of nonpositive curvature, Progress in Mathematics, vol. 61, Birkhäuser, Boston, 1985.

[2] R. Bartnik, The mass of an asymptotically flat manifold, Comm. Pure and Appl. Math. 39 (1986), 661-693.

[3] J. K. Beem, P. E. Ehrlich, and K. L. Easley, Global Lorentzian geometry, 2 ed., Pure and Applied Mathematics, vol. 202, Marcel Dekker, New York, 1996.

[4] J. K. Beem and P. E. Parker, Values of pseudo-Riemannian sectional curvature, Comm. Math. Helv 59 (1984), 319-331.

[5] G. S. Birman and K. Nomizu, The Gauss-Bonnet theorem for 2-dimensional space times, Mich. Math. Jour. 31 (1984), 77-81.

[6] E. Calabi, Oral communication, 4 April 1993.

[7] J. Cheeger and D. Ebin, Comparison theorems in Riemannian geometry, North Holland, Amsterdam, 1975.

[8] M. Dajczer and K. Nomizu, On the boundedness of Ricci curvature of an indefinite metric, Bol. Soc. Brasil Math. 11 (1980), 25-30.

[9] M. Dajczer and K. Nomizu, On sectional curvature of indefinite metrics II, Math. Ann 247 (1980), 279-282.

[10] J.-H. Eschenburg and V. Schroeder, Riemannian manifolds with flat ends, Math. Z. 196 (1987), 573-589.

[11] F. J. Flaherty, Lorentzian manifolds of nonpositive curvature, Proceedings of Symposia in Pure Mathematics, vol. 27, AMS, 1975, pp. 395-399.

[12] G. J. Galloway, Some connections between global hyperbolicity and geodesic completeness, Jour. Geom. Phys. 6 (1989), 127-141.

[13] G. J. Galloway, Space-time splitting theorems, Proceedings of the miniconference on mathematical general relativity (Canberra) (R. Bartnik, ed.), vol. 19, Australian National University, Proc. Center for Mathematical Analysis, 1989, pp. 101-119.

[14] L. Graves and K. Nomizu, On sectional curvature of indefinite metrics, Math. Ann. 232 (1978), 267-272.

[15] R. E. Greene, P. Petersen, and S. Zhu, Riemannian manifolds of faster than quadratic curvature decay, Internat. Math. Res. Notices 1 (1994), no. 9. 
[16] R. E. Greene and H. Wu, Gap theorems for noncompact Riemannianmanifolds, Duke Math. Jour. 49 (1982), 731-756.

[17] S. G. Harris, A triangle comparison theorem for Lorentz manifolds, Ind. Univ. Math. Jour. 31 (1982), 289-308.

[18] S. Hawking and G. F. W. Ellis, The large scale structure of space-time, Cambridge University Press, Cambridge, 1973.

[19] E. Heintze and $\mathrm{H}$. Karcher, A general comparison theorem with applications to volume extimates for submanifolds, Ann Scient. Éc. Norm. Sup. 11 (1978), 451-470.

[20] S. Helgason, Differential geometry, Lie groups, and symmetric spaces, Academic Press, New York, 1978.

[21] G. T. Horowitz and M. Perry, Gravitational energy cannot become negative, Phys. Rev. Lett. 48 (1982), 371-374.

[22] D. Husemoller, Fibre bundles, Springer-Verlag, 1975.

[23] H. Karcher, Riemannian comparison constructions, Global Differential Geometry (Providence R. I.) (S.-S. Chern, ed.), vol. 27, Mathematical Association of America, Providence R. I., 1989, pp. 170-222.

[24] A. Kasue and K. Sugahara, Gap theorems for certain submanifolds of Euclidean space and hyperbolic space forms, Osaka J. Math. 24 (1987), 679-704.

[25] W. Klingenberg, Contributions to Riemannian geometry in the large, Ann. Math. 69 (1959), 654-666.

[26] W. Klingenberg, Manifolds with restricted conjugate locus, Ann. Math. 75 (1963), 527-547.

[27] R. S. Kulkarni, The values of sectional curvature in indefinite metrics, Comm. Math. Helv. 54 (1979), 173-176.

[28] M. Ludvigsen and J. A. G. Vickers, A simple proof of the positivity of the Bondi mass, J. Phys. A 15 (1982), L67-L70.

[29] K. Nomizu, Remarks on the sectional curvature of an indefinite metric, Proc. AMS 89 (1983), 473-476.

[30] T. Parker and C. Taubes, Witten's proof of the positive mass theorem, Commun. Math. Phys. 84 (1982), 223-238.

[31] O. Reula and K. P. Tod, Positivity of the Bondi energy, J. Math. Phys. 25 (1984), 1004-1008. 
[32] R. Schoen and S. T. Yau, On the proof of the positive mass conjecture in general relativity, Comm. Math. Phys. 65 (1979), 45-76.

[33] R. Schoen and S. T. Yau, Proof that the Bondi mass is positive, Phys. Rev. Lett. 48 (1982), 369-371.

[34] R. Schoen and S. T. Yau, Conformally flat manifolds, Kleinian groups and scalar curvature, Invent. Math. 92 (1988), 47-71.

[35] V. Schroeder and W. Ziller, Local rigidity of symmetric spaces, Trans. Ams. Math. Soc. 320 (1990), 145-160.

[36] N. Steenrod, The topology of fibre bundles, Princeton University Press, Princeton, New Jersey, 1951.

[37] V. A. Toponogov, Computation of the length of a closed geodesic on a convex surface, Dokl. Akad. Nauk. SSSR. 124 (1959), 282-284.

[38] R. Wald, Introduction to general relativity, University of Chicago Press, Chicago, 1984.

[39] E. Witten, A simple proof of the positive energy theorem, Commun. Math. Phys 80 (1981), 381-402.

[40] J. A. Wolf, Spaces of constant curvature, 3 ed., Publish or Perish Inc., 1974.

Received December 6, 1996.

Royal Institute of TECHNOLOGY

S-100 44 STOCKHolm, SWEdEN

E-mail: larsa@math.kth.se

AND

University of South Carolina

Columbia, S.C. 29208, USA

E-mail: howard@math.sc.edu 